VILMOS ÁGEL

\title{
IST DIE DEPENDENZGRAMMATIK WIRKLICH AM ENDE?
}

\author{
Valenzrealisierungsebenen, Kongruenz, Subjekt und die Grenzen des \\ syntaktischen Valenzmodells*
}

0. Dependenzgrammatik und/oder Rektions- und Bindungstheorie?

0.1. Zur Vergleichbarkeit

0.2. Zur Adäquatheit

1. Realisierungsebenen der Valenz

1.1. Das traditionelle Kongruenzargument

1.2. Kongruenz und Rektion - Begriffsbestimmungen mit Peter Eisenberg

1.3. Eine dependenzgrammatische Forschungslinie: Grenzen des syntaktischen Valenzmodells und Möglichkeiten eines Zwei-Ebenen-Modells

1.4. Zum Verhältnis der Ebenen im Zwei-Ebenen-Modell

1.5. Ein Drei-Ebenen-Modell?

2. Es impersonale und das Zwei-Ebenen-Modell

2.1. Andeutung des es-Problems

2.2. Zum Status des es

3. Subjekt und das Zwei-Ebenen-Modell

4. Kongruenz, Rektion und das Zwei-Ebenen-Modell

4.1. Kongruenzverletzungen: ein rein formales Problem?

4.2. Ein Wort zum Diskurs

4.3. Ein Wort zum „Kopula“-Problem

5. Ellipsen und das Zwei-Ebenen-Modell

5.1. Zum Status grammatischer Ellipsen

5.2. Zum Status lexikalisch-pragmatischer Ellipsen

6. Standardsprache und das Zwei-Ebenen-Modell

7. Schlußbemerkungen

0. Dependenzgrammatik und/oder Rektions- und Bindungstheorie?

\subsection{Zur Vergleichbarkeit}

Heide Wegener (1990) vergleicht die Leistung der Dependenzgrammatik (im weiteren: DG) mit der der Rektions- und Bindungstheorie (im weiteren: GB) und kommt zu einem in allen von ihr untersuchten Punkten eindeutigen Schluß: DG - gewogen und zu leicht befunden.

Der vorliegende Aufsatz, der dependenzgrammatisch orientiert ist und sich mit den Möglichkeiten eines nicht rein syntaktischen strukturellen Valenzrealisierungsmodells (kurz: Valenzmodells) befaßt, kann in dem Sinne auch als ein Diskussionsbeitrag verstanden werden, als er die Arbeit von Wegener

* Vorliegender Beitrag wurde im Rahmen eines Forschungsstipendiums der Alexander von Humboldt-Stiftung fertiggestellt. An dieser Stelle sei der AvH für ihre Unterstützung gedankt. 
sozusagen zum Anla $ß$ nimmt, um auf wichtige Überlegungen, die im dependenzgrammatischen Rahmen angestellt wurden, aufmerksam zu machen. Ein systematischer „Gegenvergleich“ kommt allerdings aus mindestens zwei Gründen nicht in Frage:

(1) Trotz der verdienstvollen Bemühungen von Wegener muß man prinzipielle Vorbehalte gegenüber der Vergleichbarkeit von Theorien anmelden. Bekanntlich sind es nämlich die Theorien selbst, die ihren Gegenstand schaffen. Daraus, daß „ein direkter Vergleich mit den Objekten erkenntnistheoretisch unmöglich (ist)" (Heringer (1970), 59), folgt, daß Theorien rein holistisch gesehen, wenn also ihre Begriffe und Kategorien als bloße (nichtsubstanzielle) Zeichen-Valeurs eines Systems (im Saussureschen Sinne) gegenübergestellt werden sollen, unvergleichbar sind (Mudersbach (1990), 94). Hinzu kommt noch m. E., daß die interne Wohldefiniertheit zweier Theorie-Systeme, d. h. der Grad des gegenseitigen Angewiesenseins von theorieinternen Begriffen und Kategorien zweier Theorien, nicht identisch sein kann. (Sonst wären sie ja nur Notationsvarianten derselben Theorie). Demnach gibt es eher „naturwissenschaftlich" und eher "geisteswissenschaftlich" organisierte Theorien. Bei den ersteren wird die Art von sog. normaler wissenschaftlicher Arbeit, die Kuhn $(1976,47)$ die „gegenseitige Anpassung von Fakten und Theorie“ genannt hat, eine bedeutendere Rolle spielen als bei den letzteren, was sowohl seine Vorteile (Strenge der Denkweise: neue Fakten werden aus allen möglichen Perspektiven durchleuchtet) wie auch seine Nachteile (Rigidität der Theorie: mit Anwachsen der Faktenmenge und Ausbau der Theorie kommt es zu immer größeren Anpassungsschwierigkeiten) hat. Die Rolle dieser gegenseitigen Anpassung in der GB scheinen auch Grewendorf/Hamm/Sternefeld $(1987,188)$ durch den Hinweis hervorzuheben, „daß auf der Ebene der Beobachtungsadäquatheit nicht einzelne Regeln ,richtig' oder ,falsch' sind, sondern erst die Grammatik als ganze. Daher lassen sich einzelne Regeln nur in bezug auf andere Regeln rechtfertigen, nicht in bezug auf die empirischen Daten allein." Die erwähnten Anpassungsschwierigkeiten, der Konflikt zwischen „empirischen Daten“ und „Regeln“, sind quasi vorprogrammiert, wobei man hinzufügen muß, daß nichtfunktionale Erklärungen in gewisser Hinsicht immun gegen Widerlegungen sind (vgl. von Stechow/Sternefeld (1988), 20f.).

Die GB definiert sich nun als eine Theorie der sog. mentalen Grammatik, des Sprachvermögens. Und da als der Gegenstand der DG am ehesten wohl die sog. objektive Grammatik, d. h. das „dem Objekt Sprache (einer Einzelsprache) selbst innewohnende[.] Regelsystem, unabhängig von dessen Beschreibung durch die Linguistik und von dessen Beherrschung durch den Sprecher" (Helbig (1988), 160), angegeben werden kann, scheint der grundlegende Unterschied zwischen den Gegenständen beider Theorien in der Opposition, intern (mental) vs. extern (objektiv) ${ }^{6}$ zu liegen. ${ }^{1}$ Aus dem erwähnten straffen Theo-

1 Zu den Begriffen ,objektive, psychologische (mentale), linguistische Grammatik` vgl. z. B. Helbig (1988), $160 \mathrm{f}$., zu den entsprechenden Begriffen der GB ,E-Sprache (EStruktur) ${ }^{6}$ und ,I-Sprache (I-Struktur) vgl. etwa Fanselow/Felix (1990), 40 ff. 
rieaufbau der GB (vgl. auch 0.2.) folgt allerdings, daß Theorien der objektiven Grammatik wahrscheinlich und paradoxerweise mindestens genauso „intern (mental)" sind wie die GB, die Unterscheidung zwischen mentaler und objektiver Grammatik (einer Einzelsprache) verflüchtigt sich also weitgehend (vgl. auch von Stechow/Sternefeld (1988), 29). Demnach ist der Unterschied eher in den Grammatik- bzw. Sprachbegriffen zu suchen:

Die GB untersucht die Grammatik, einen für sie biologischen Gegenstand. Die Sprache als soziales Gebilde, ein „Epiphänomen“ (von Stechow/Sternefeld (1988), 28), bleibt unberücksichtigt. Die DG untersucht dagegen die Grammatik, ein soziales Teilgebilde des sozialen Gebildes ,Sprache‘ (= ,Einzelsprache'). Und da die GB eine insgesamt "naturwissenschaftlicher" organisierte Theorie ist als die sich im Anschluß an Tesnière entwickelnde sog. empirische Richtung der DG (Heringer/Strecker/Wimmer (1980), 167), um die es in der vorliegenden Arbeit geht, wäre eine Vergleichsgrundlage in der Tat nicht einfach zu etablieren. ${ }^{2}$ Als Beispiel denke man nur an die "Subjekte" beider Theorien: an den Spezifikator des X-bar-theoretischen funktionalen Kopfes INFL der GB (Olsen/Fanselow (1991), 4; vgl. auch 1.4.2.2. und 1.4.4.) und den Erstaktanten Tesnières.

Trotz (oder wegen?) der grundsätzlichen Unvergleichbarkeit von Theorien muß man sich jedoch zum scheinbaren Paradoxon bekennen, daß Vergleiche (besser: Gegenüberstellungen) von Theorien einen hohen Erkenntniswert haben können, vorausgesetzt, man ist sich der eigentlichen (= holistischen) Unvergleichbarkeit stets bewußt und zieht aus ihr immer die notwendigen Konsequenzen. Dabei ist das Paradoxon aus zwei Gründen nur scheinbar:

(a) weil es theoretisch möglich ist, zwei Begriffe (Kategorien) aus zwei Theorien anhand eines für die Zwecke der Untersuchung ausgewählten Ausschnitts ihrer jeweiligen inhaltlichen Vernetzungen mit anderen Begriffen (Kategorien) der eigenen Theorie gegenüberzustellen und so einen sog. hol-atomistischen Vergleich (Mudersbach (1983) und (1990)) anzustellen und

(b) weil es in jeder Theorie verhältnismäßig theorieneutrale oder neutralisierbare Lösungen gibt, die dementsprechend in andere Theorien integriert werden können. ,Die notwendigen Konsequenzen ziehen' heißt gerade, daß diese Integrierbarkeit von Fall zu Fall überprüft werden muß (vgl. 1.4.4.).

(2) Der andere Grund, auf einen systematischen „Gegenvergleich“ zu verzichten, ist, da $\beta$ in dem vorliegenden Aufsatz die Dependenzgrammatik teilweise auf den Valenzansatz eingeschränkt wird.

${ }^{2}$ Im übrigen ist es die generative Grammatik, in der der Anspruch auf einen eigenen Untersuchungsgegenstand schon immer stark betont wurde: „Generative grammar is sometimes referred to as a theory [...]. In fact, it is not a theory any more than chemistry is a theory. Generative grammar is a topic, which one may or may not choose to study.“ (Chomsky (1986), 4) 


\subsection{Zur Adäquatheit}

In ihrer vergleichenden Untersuchung wendet Wegener (1990, $152 \mathrm{f}$.) alle drei in der Theorie der generativen Grammatik entwickelten Bewertungskriterien für Grammatikbeschreibungen (Beobachtungs-, Beschreibungs- und Erklärungsadäquatheit) auf beide Theorien an. Dieses Vorgehen ist jedoch nicht unproblematisch, denn die generativistischen Bewertungskriterien können, sind einmal die unterschiedlichen Gegenstände der Theorien akzeptiert, nicht auf jede beliebige Grammatiktheorie übertragen werden:

(1) Daraus, daß der Untersuchungsgegenstand der DG ein soziales Teilgebilde einer Einzelsprache ist, folgt, daß das generativistische Kriterium der Erklärungsadäquatheit nicht auf die DG angewendet werden kann. Ein funktionalistisches Kriterium der Erklärungsadäquatheit macht das konkrete Sprechen zum Maßstab abstrakter Strukturen (Coseriu (1988)), kann daher nicht mit einem autonomistischen identisch sein. Orientiert man sich an Rudi Kellers (1990, 48) Erklärungsprinzip („Die Stärke einer Erklärung wächst mit der Schwäche der vorausgesetzten Annahmen."), so kann man die funktionalistische Annahme, daß das konkrete Sprechen der abstrakten Sprachfähigkeit logisch vorgeordnet ist, daß also die abstrakte Sprache um des konkreten Sprechens willen da ist und nicht umgekehrt, m. E. als schwach einstufen.

(2) Was das Kriterium der Beobachtungsadäquatheit anbelangt, ist die Forderung nach einer „richtigen“, nur „grammatische“ Sätze zulassenden Beschreibung eine Selbstverständlichkeit. Da jedoch ,Grammatikalität" nur in bezug auf eine Grammatiktheorie und ,Angemessenheit/Akzeptabilität' nur in bezug auf das konkrete Sprechen interpretierbar sind, scheint ein auf theorieunabhängigen Kriterien beruhender Begriff der „Richtigkeit“ - wenn überhaupt, dann - nur funktional möglich zu sein.

(3) Noch problematischer ist das Kriterium der Beschreibungsadäquatheit. Nach Wegener sollten „vorgeschlagene Regeln und Strukturen“ „unserer Intuition“ (1990, 152) entsprechen. Dabei ist unklar, was genau mit „unserer“ angesprochen wird. Auf eine kollektive Intuition dürfte es sich kaum beziehen, denn eine solche gibt es nicht. Des weiteren kann eine Intuition unmittelbar nur auf das soziale „Epiphänomen“ ,Sprache“, nicht jedoch auf die mentale Grammatik bezogen werden. Ob und wie sie von diesem „sozialen Touch“ befreit werden könnte, ist fragwürdig. Und es ist gerade diese Unklarheit des Intuitionsweges zwischen Sprache (bzw. Angemessenheit) und Grammatik (bzw. Grammatikalität), die einen methodologischen Schatten auf die GB wirft. Da sie nämlich immer zwei Fliegen - eine universalgrammatische und eine einzelsprachliche - mit einer Klappe - mit demselben Grundbestand von Erklärungen/Beschreibungen - schlagen will (bzw. wegen ihrer „Naturwissenschaftlichkeit" auch schlagen wollen muß), müßte der GB-Syntaktiker immer Rechenschaft darüber ablegen, warum in den jeweiligen Erklärungsund/oder Beschreibungszusammenhang die Daten einbezogen werden, die er gerade einbezieht, und ob der Erklärungs- und/oder Beschreibungszusammen- 
hang wirklich relevant ist. Die „natürlichen inneren Widersprüche jedes realisierten Sprachsystems“ (Coseriu (1974), $110 \mathrm{ff}$.) machen jedes Verfahren problematisch, das Daten aus solchen ,geschichtlich gewachsenen“ Gefügen von einzelsprachlichen Widersprüchen einfach herauslöst und sie ohne nähere Begründung in einen (und denselben) geschichtsgebunden-geschichtsentbundenen (oder allgemeiner: kulturgebunden-kulturentbundenen) Erklärungsund/oder Beschreibungszusammenhang stellt. Und eine Theorie ohne begründete Datengrundlage, d. i. ohne ein transparentes Verhältnis zwischen Daten und Untersuchungsgegenstand (Untersuchungsgegenständen), scheint mir eine contradictio in adjecto zu sein (vgl. auch Comrie (1989), 5 ff.).

Demgegenüber besteht ein Vorteil der DG in dem transparenten Verhältnis zwischen Daten und Untersuchungsgegenstand. Soweit das Konzept ,Einzelsprache' (und/oder ,funktionelle Sprache', vgl. z. B. Coseriu (1988), 24 ff.) nicht grundsätzlich in Frage gestellt wird und soweit die Daten nachweislich der gerade untersuchten Einzelsprache (und/oder funktionellen Sprache) entstammen, scheint der diesbezügliche methodologische Hintergrund gesichert zu sein.

Nun geht es hier nicht um eine umfassende Kritik des Kriteriums der Beschreibungsadäquatheit, sondern um Vorüberlegungen einer dependenziell orientierten Arbeit, deren Verfasser die Folgen der methodologischen Unklarheiten einer anderen Theorie nicht tragen möchte. Deshalb beschränke ich mich auf die folgenden Bemerkungen:

(a) Intuitivität ist nicht meßbar. Aber selbst, wenn wir Kriterien hätten, nach denen wir konkurrierende Regelvorschläge (Strukturenvorschläge) auf einer Skala ab- und zunehmender Intuitivität anordnen könnten, müßten wir angesichts der per-definitionem-Individualität von Intuitionen damit rechnen, daß die Urteile unterschiedlich ausfallen. Im Falle eines Gesamturteils würden wir folglich im Bereich der Statistik landen.

(b) Arbeitet man als Generativist trotzdem mit der Beschreibungsadäquatheit als einem zwar definier-, aber nicht praktizierbaren Kriterium, kommt man mit der Theorieentwicklung immer wieder in die Situation, daß man Lösungen im Namen einer angenommenen größeren Erklärungsadäquatheit aufgibt, ohne sich eigentlich sicher sein zu können, ob die neue Lösung beschreibungsadäquater ist als die alte, was natürlich auch mit der erwähnten Ungeklärtheit der Datengrundlage zu tun hat. Zwar impliziert nach generativistischer Áuffassung die Erklärungs- die Beschreibungsadäquatheit, überzeugen könnte man sich von dem empirischen Gehalt der Beschreibungsadäquatheit aber nur dann, wenn die „höhere“ Intuitivität einer vorgeschlagenen Regel oder Struktur auf unabhängigen Kriterien beruhen würde. Bezieht man das Implikationsverhältnis nicht auf einzelne Regeln, sondern auf die zu erforschende mentale Grammatik (Universalgrammatik und/oder mentale Einzelgrammatiken) insgesamt, ist die ,reine“ Überprüfung einzelner Regeln auf ihre Beschreibungsadäquatheit ebensowenig möglich wie die auf ihre Beobachtungsadäquatheit (vgl. 0.1.). Eine theorieunabhängige Intuition ist in der Wissenschaft eine Illusion. 
(c) Ich gehe davon aus, daß kein Dependenzgrammatiker kontraintuitive Entscheidungen bewußt anstrebt. Insofern, schiebt man einmal alle grundsätzlichen Probleme beiseite, reduziert sich das Intuitionsproblem auf die Fragen, (c1) welcher theoretische Rahmen dem Forscher mehr „kanonische“ Beschränkungen auferlegt bzw. (c 2) welche Theorie die „besseren“ Forscher hat.

Auf (c 2) läßt sich keine sinnvolle Antwort geben. Was (c1) anbelangt, ist eine quantitative Antwort ohnehin unmöglich. Eine qualitative ist angesichts der unterschiedlichen Theoriedimensionen und theorieinternen Zwänge (vgl. 0.1. und (d)) ebenfalls in Frage zu stellen. Was bleibt, ist, daß man versucht, für einen begrenzten Phänomenbereich Lösungen anzubieten, denen der Leser ansieht, daß sie in erster Linie nicht theorieinternen Dogmen - die anderweitig sehr wohl begründet sein können - gerecht werden wollen. Im Grunde genommen sind wir also zu der Schwerpunktsetzung bei der normalen wissenschaftlichen Arbeit (Kuhn (1976), 37 ff.) und damit zu dem methodologischen Grundproblem zurückgekehrt, zu dem Rudi Keller mit dem oben zitierten Satz Stellung bezieht.

(d) Schließlich eine rhetorische Frage: Verhält es sich nicht mit jeder Theorie zwangsweise so, daß allem Anschein nach intuitionskonforme Entscheidungen an einer Stelle der Beschreibung (Erklärung) intuitionsnonkonforme Entscheidungen an anderen Stellen erzwingen?

Statt Beschreibungsadäquatheit, die also eher ein „privates Barometer“ des Forschers als ein Kriterium darstellt, möchte ich das Kriterium der soziolinguistischen Integrierbarkeit vorschlagen. Demnach ist ein systemlinguistisches Modell adäquat zu nennen, wenn es in adäquate soziolinguistische Modelle - inklusive Sprachwandeltheorien - integrierbar ist (vgl. 6.).

Ans Ende des Adäquatheitsabschnittes soll eine verallgemeinerungswürdige Bemerkung über die Regel bewege- $\alpha$ der GB gestellt werden: „Die Regel ist von einer geradezu lächerlichen Einfachheit. Transformationsgrammatiker aus der Zeit der späten fünfziger Jahre wären in Gelächter ausgebrochen, hätte man ihnen diese Regel als die zentrale Transformationsregel vorgestellt." (von Stechow/Sternefeld (1988), 96) Müßte man denn angesichts der Vorhersagbarkeit ähnlicher ,epochenübergreifender“ Gelächter über unsere gesamte wissenschaftliche Tätigkeit bei der Verkündung von Adäquatheitsmaßstäben nicht vorsichtiger sein?

\section{Realisierungsebenen der Valenz}

\subsection{Das traditionelle Kongruenzargument}

Wegeners Überlegungen erstrecken sich auf vier Themenkomplexe, von denen einer „Die Behandlung des Subjekts“ (1990, 153 ff.) darstellt. Hier führt sie fünf Argumente an, die dagegen sprächen, daß das Subjekt dem Verb untergeordnet wird. Aus thematischen und Raumgründen soll hier nur das erste, die Kongruenz, behandelt werden: „Das Subjekt steht durch die Kon- 
gruenz mit dem Verb auf einer Stufe mit diesem, also neben und nicht unter ihm, denn aufgrund der Kongruenz besteht zwischen beiden nicht Dependenz, sondern Interdependenz.“ $(1990,153)$ Um dieses Argument bewerten zu können, muß zunächst (a) die allgemeine Redeweise von der Kongruenz differenziert und (b) auf die Realisierungsebenen der Valenz eingegangen werden.

\subsection{Kongruenz und Rektion - Begriffsbestimmungen mit Peter Eisenberg}

Um die allgemeine Redeweise von der Kongruenz zu differenzieren, soll Peter Eisenbergs Begrifflichkeit herangezogen werden. Er unterscheidet u. a. die syntagmatischen Relationen ,Rektion“ und ,Kongruenz': „Eine Konstituente $f_{1}$ regiert eine Konstituente $f_{2}$, wenn die Form von $f_{2}$ durch eine Paradigmenkategorie von $\mathrm{f}_{1}$ festgelegt ist [...].“ $(1989,52)$ „Eine Konstituente $f_{1}$ kongruiert mit einer Konstituente $f_{2}$, wenn $f_{1}$ bezüglich mindestens einer Einheitenkategorie von einer Einheitenkategorie von $f_{2}$ abhängt." $(1989,55)$ Dabei werden durch eine Einheitenkategorie paradigmatisch zusammengehörige Wortformen intern gegliedert, während eine Paradigmenkategorie allen Formen eines Paradigmas gemeinsam ist. Beispielsweise ist ,Genus' eine Einheitenkategorie beim Artikel, ,Maskulinum' eine Paradigmenkategorie aller maskulinen Substantive (vgl. Eisenberg (1989), 36 ff.; vgl. auch Lehmann (1982), 207). Demnach können folgende syntagmatische Relationen zwischen (syntaktischem) Subjekt und Finitum festgestellt werden (1-3 in Anlehnung an Eisenberg):

(1) Da ,Person' sowohl eine Einheitenkategorie des sog. Personalpronomens wie auch eine (andere, aber gleichgenannte) des Verbs ist, gibt es zwischen ihnen eine Personenkongruenz.

(2) Da nichtpronominale (substantivische) Nomina über die Paradigmenkategorie ,3. Person' verfügen, ${ }^{3}$ regieren sie in Subjektposition das Verb hinsichtlich Person.

(3) Da ,Numerus' sowohl eine Einheitenkategorie des Nomens (Substantiv und Personalpronomen) wie auch eine (andere, aber gleichgenannte) des Verbs ist, gibt es zwischen ihnen eine Numeruskongruenz.

(4) Aus der Eisenbergschen Unterscheidung zwischen Einheiten- und Paradigmenkategorien bzw. aus (1) - (3) kann man eine weitere Konsequenz in bezug auf das deutsche Verb ziehen. Vergleicht man Sätze des Typs *Mich $\left(/{ }^{*}\right.$ Meiner $/{ }^{*}$ Mir $)$ gehe, ${ }^{*}$ Dich $\left(/{ }^{*}\right.$ Deiner $/{ }^{*}$ Dir $)$ gehst mit ihren korrekten Pendants Ich gehe, Du gehst, stellt man fest, daß in allen Fällen die Kongruenz sowohl hinsichtlich Person als auch hinsichtlich Numerus gewährleistet ist. Die Inkorrektheit der mit * versehenen Sätze kann daher nur von der Verlet-

${ }^{3}$ Sie sind ersetzbar durch er, sie, es, haben jedoch keine Formen in 1. und 2. Person (vgl. Eisenberg (1989), 286 f.). 
zung einer syntagmatischen Relation hinsichtlich des Kasus herrühren. ${ }^{4}$ Da ,Kasus‘ eine Einheitenkategorie des Nomens, jedoch keine des Verbs ist, können die Inkorrektheiten im vorgegebenen methodischen Rahmen nur damit erklärt werden, daß eine Rektionsbeziehung verletzt wurde. Dieses Verb muß also das Nomen genau in dem Sinne regieren, daß, Nominativ‘ eine Paradigmenkategorie der finiten Formen von geh-ist. Allgemeiner: Zwischen einem deutschen Finitum und einer oder mehreren seiner kasuellen Ergänzungen gibt es Rektionsbeziehungen hinsichtlich des Kasus. ${ }^{5}$ Auf die Nominativrealisierung wird ausführlicher in 1.4.2.2. einzugehen sein.

Aufgrund von (1)-(4) kann man nicht mehr pauschal behaupten, die Kongruenz (im undifferenzierten Sinne) begründe eine interdependente Beziehung zwischen Subjekt und Verb (vgl. auch Welke (1992)). Bei der Personenund Numeruskongruenz käme eine Interdependenz nur dann in Frage, wenn die Realisierungsbedingungen der korrelierenden Einheitenkategorien von Personalpronomen und Verb bzw. Nomen und Verb völlig identisch wären. Wie noch zu zeigen sein wird, sind diese jedoch von den strukturellen Realisierungsbedingungen von Aktanten nicht unabhängig. Und letztere sind für Nomen und Verb nicht einmal im hinsichtlich der strukturell-typologischen Realisierungsvarianz sehr rigiden Deutsch vollkommen parallel (vgl. 1.3.5. und 1.3.7.).

Die Personenrektion des substantivischen Subjekts und die Kasusrektionen des Verbs begründen per definitionem (entgegengesetzt gerichtete) dependente Beziehungen.

Abschließend sei noch darauf hingewiesen, daß es in der Dependenzgrammatik durchaus möglich (und üblich) ist, Interdependenz als einen Spezialfall von Dependenz zu behandeln. Eine interdependente Beziehung zwischen zwei Kategorien wie Subjekt und Prädikat oder eine interdependente Vorkommensrelation wie die zwischen ich und gehe in ich gehe sind methodisch einwandfrei beschreibbar als die logische Konjunktion von zwei dependenten Kategorien oder Vorkommensrelationen (zu diesem Problemkomplex vgl.

${ }^{4}$ Verständlicherweise spricht keine der mir bekannten traditionellen Grammatiken von Kasuskongruenz, schließlich erkennen sie (implizite) nur Einheitenkategorien an. Daraus folgt jedoch, daß man über die Inkorrektheit dieser Sätze keine Rechenschaft ablegen kann.

${ }^{5}$ Hier wird besonders deutlich, daß die traditionelle Gleichbenennung von grammatischen Kategorien bei verschiedenen Wortarten nur eine irreführende Tradition darstellt (vgl. Eisenberg (1989), 286). Denn während ,Kasus' beim deutschen Nomen eine Einheitenkategorie mit vier Realisierungstypen ist, ist ,Kasus' beim deutschen Verb ein Oberbegriff für alle möglichen Kombinationen von Kasusformen, deren jede eine eigene Paradigmenkategorie darstellt. Z. B. haben die finiten Formen von geh- die Paradigmenkategorie ,Nominativ', die von vergeud- die Paradigmenkategorien ,Nominativ' und ,Akkusativ' usw. Kasuskategorien bei Verben anzunehmen, überlegt auch Matthews (1981), 249. In der GB wird die Kasuszuweisung im Rahmen der Kasustheorie diskutiert (vgl. z. B. Fanselow/Felix (1990 a), 62 ff.; s. auch 1.4.2.2. und insb. Anm. 27). 
Heringer/Strecker/Wimmer (1980), 167 ff.). Daher kann die Gegenüberstellung am Ende des Wegener-Zitats (,[...] nicht Dependenz, sondern Interdependenz.") von dieser Interpretation aus ebenfalls nicht als Teil eines (Gegen)Arguments gedeutet werden.

\subsection{Eine dependenzgrammatische Forschungslinie: Grenzen des syntaktischen Valenzmodells und Möglichkeiten eines Zwei-Ebenen-Modells}

Es gibt innerhalb der Dependenzgrammatik eine leider wenig beachtete Forschungslinie, deren Wurzeln bis Tesnière zurückreichen und deren Vertreter sich nicht wie die der Hauptströmung der empirischen DG mit den verschiedenen Ebenen der Valenz als Virtualität bzw. den grammatischen und kontextuell-situationellen Bedingungen ihrer Realisierung befassen, sondern mit ihren strukturell-typologisch bedingten Realisierungsebenen. Diese Forschungsrichtung ist $u$. a. deshalb vielversprechend, weil sie den Valenzbegriff für typologische und konfrontative Untersuchungen zugänglich macht. Im folgenden möchte ich wichtige Stationen dieser Forschungslinie nachzeichnen, um ihre Ergebnisse zu diskutieren und einige Vorschläge zu machen.

1.3.1. Daß selbst Numerus- und Personenkongruenz zwischen Personalpronomen und Finitum bezüglich der Realisierungsebenen des Erstaktanten keine symmetrischen Relationen darstellen, zeigt als erster Lucien Tesnière (1959, $84 \mathrm{f}$.). Gehe man nämlich davon aus, daß eine Form wie (nous) aim-ons aus dem zugrundeliegenden Stamm aim- abgeleitet wird, so müsse man die Endung -ons als Index auffassen, da ja der Stamm weder die Kategorie ,Person' noch die Kategorie ,Numerus ${ }^{6}$ ausdrückt. ${ }^{6} \mathrm{Da}$ die Funktion des persönlichen Index (d. h. etwa: des nichtemphatischen Personalpronomens) ebenfalls die Anzeige von Person und Numerus beim Verb ist, liegt es auf der Hand, daß Tesnière die persönlichen Indizes des Französischen als „''équivalent syntaxique exact“ $(1959,85)$ der Personalendungen etwa des Lateinischen auffaßt: „La seule différence est que l'indice est postposé et agglutiné en latin, tandis qu'il est préposé et autonome (au moins dans l'orthographe) en français.“ (ebd.)

Aus dieser integrativen Sicht der Betrachtung der Verbmorphologie zusammen mit der syntaktischen Umgebung des Finitums folgen zahlreiche Entfaltungs- und Anwendungsmöglichkeiten des Modells, von denen schon Tesnière viele andeutet. Was die Kongruenz anbelangt, folge aus ihr, da $\beta$ die Subjekt-Prädikat-Grenze nicht einmal durch eine Wortgrenze markiert sei, denn in einem Satz wie filius amat patrem gehöre die Endung - $t$ zum Subjekt. $(1959,104)$ Die Kongruenz begründet also nicht irgendeine exklusive und symmetrische Beziehung zwischen Subjekt und Prädikat, sondern sie verwischt die Wortgrenze zwischen beiden, was „s'accorde mal avec l'opposition de ces

${ }^{6}$ Die andere Möglichkeit nach ihm ist, einer Verbendung den Wert eines Translativs zuzuschreiben. In diesem Falle muß man aber eine Verbform (etwa Sg. 1. P.) als zugrundeliegend definieren. 
deux notions, tandis qu'elle ne fait aucune difficulté dans l'hypothèse du nœud verbal comme nœud central.“ (ebd.) Im 61. Kapitel folgt dann der Schlüsselsatz, der m. E. als Motto der ganzen Forschungslinie gelten könnte: „Dès lors parler de l'accord entre le verbe et son prime actant revient simplement à constater que le verbe a déjà incorporé un prime actant de fonction syntaxique identique au prime actant actuellement vivant.“ $(1959,139)$ Nach Seyfert $(1976,16 \mathrm{f}$.) enthält die - nennen wie sie so - Kongruenzthese Tesnières „eine kühne These über die verbale Konjugation, daß nämlich die Flexionsendung der 1. Pers. Teil des Subjekts (und nicht des Prädikats) sei. Dem steht seine eigene Ansicht entgegen, wonach die Fragen der Morphologie den strukturellen, syntaktischen Fragen nachgeordnet seien [...].“ Auf diese wichtige Bemerkung müssen wir noch zurückkommen.

Tesnière sieht die Konsequenzen für den typologischen Vergleich ebenfalls ganz deutlich. Einem lat. ille amat entspreche z. B. nicht frz. il aime, sondern lui, il aime, denn als Personen- und Numerusindex funktioniere - $t$ allein im Lateinischen. $(1959,140)$ Das von Sprache zu Sprache unterschiedliche Verhältnis von Personalpronomen und Verbmorphologie (inklusive 0-Pronomen und 0-Morphologie) bei der Anzeige von syntagmatischen Relationen kann also einer Sprachtypologie zugrunde gelegt werden. Tesnière geht sogar noch weiter, indem er seine integrative Sichtweise auch auf den Zweitaktanten objektiv (definit) konjugierter ungarischer Verben ausdehnt und feststellt, ,que le verbe s'agglutine le second actant pour en faire également une désinence personnelle." $(1959,141)$ Der Zweitaktant ist also bereits in der Verbmorphologie angelegt, d. h. das ungarische Verb hat - im Gegensatz zum deutschen - sowohl Einheitenkategorien ,Kasus' (,Nominativ' und ,Akkusativ') als auch Paradigmenkategorien ,Kasus' (alle anderen Kasusformen), da es im Ungarischen ein Kasusparadigma aus zwei Gruppen von Verbformen gibt: Verbformen mit inkorporiertem Erstaktanten (wie lat. cantat) und Verbformen mit inkorporiertem Erst- und Zweitaktanten (mehr dazu in 1.3.7.).

Zusammenfassend können die - wie ich glaube - revolutionären Gedanken Tesnières wie folgt auf das Kongruenzproblem bezogen werden: Die Kongruenz- und Rektionsrelationen zwischen (syntaktischem) Subjekt und finitem Verb drücken nicht bzw. nicht nur Beziehungen zwischen zwei Konstituenten des Satzes aus, sondern auch solche zwischen zwei Realisierungsebenen derselben Konstituente. ${ }^{7}$ Eine universell gültige Zweiteilung des Satzes in Subjekt und Prädikat auf Kongruenzbasis scheint hier nicht nur in Anbetracht der Umdefinierung des Konstituentenbegriffes durch Tesnière in Frage gestellt, sondern auch, weil es syntagmatische Relationen zwischen den sagen wir - nichterstaktantenbezogenen Realisierungsebenen derselben Konstituente gibt und weil die Art dieser Relationen von Sprache zu Sprache unterschiedlich sein kann (vgl. 1.3.7. und 1.4.). Dabei ist bei weitem nicht die

${ }^{7}$ Dabei ist es unerheblich, ob man die Quelle der Kongruenz im Nomen oder im Verb erblickt. 
definite Konjugation des Ungarischen das einzige Beispiel. Gerade in der generativistischen Literatur - im Rahmen der Erörterung des sog. pro-dropParameters - bzw. in der Allgemeinen Sprachwissenschaft - u. a. im Rahmen von dependenzbezogenen Typologien (Pasierbsky (1981); Mosel (1984); Nichols (1986), $61 \mathrm{ff}$.) - werden exotische und weniger exotische Sprachen wie Abchasisch, Arabisch, Baskisch, Georgisch, Hebräisch, Navaho, Swahili, Tok Pisin, Warlpiri und viele andere mehr behandelt. In der Allgemeinen Sprachwissenschaft kann das Problem bis hin zu den amerikanischen Altmeistern Boas und Bloomfield zurückverfolgt werden. ${ }^{8}$

1.3.2. Wie oben durch das Seyfert-Zitat schon angedeutet wurde, enthält Tesnières These über Konjugation einen Widerspruch. Er geht vom Primat der Syntax gegenüber der Morphologie aus, dabei analysiert er Sprachen, in denen ein Finitum einen kompletten Satz darstellen kann. Diesem Widerspruch versucht Harald Weinrich (1967, $110 \mathrm{ff}$.) abzuhelfen, indem er eine einpolige Satzanalyse (illustriert am Baudelaire-Textstück les hommes d'équipage prennent des albatros) vorschlägt (111):

„Im Mittelpunkt des Satzes steht das Verb, das von einer Schicht von paradigmatisch geordneten, ,gebundenen' Morphemen (,bound forms') umgeben ist, ohne die es gar nicht vorkommen könnte: einem Morphem zur Angabe der handelnden Person, dem Signal für Tempus, einem Morphem für die Assertion, also für die Bejahung oder Verneinung. Das Akkusativ- und das Dativobjekt können ebenfalls durch gebundene Morpheme repräsentiert werden [...]“

Die Funktion dieser innersten Schicht der Morpheme am Verb sei, das Verb auf die Sprechsituation, d. h. auf die drei elementaren Rollen der Kommunikation, zu beziehen.

„Die innerste Schicht der Morpheme, die das Verb umgeben, kann nun expandiert werden. Wenn ich z. B. das Verb habe ils prennent, so kann ich ils expandieren zu dem, was wir Subjekt nennen: les hommes d'équipage, und ils fällt dann weg. Sie sehen hier sehr deutlich, wie sich diese Analyse von der klassischen Analyse nach Subjekt und Prädikat unterscheidet; das, was wir Subjekt nennen, ist jetzt nicht einer der Pfeiler des Satzes, sondern die semantische Expansion eines Morphems am Verb, eines Morphems unter einigen anderen." (111 f.) ${ }^{9}$

Zwar ist die Analyse Weinrichs im Vergleich zu der elaborierten Untersuchung Tesnières eher als eine Ideenskizze anzusehen, sie enthält trotzdem einen weiterweisenden Gedanken: Der Satz wird als eine schichtenweise Expansion der Morpheme am Verb verstanden. Wohl könnte man seine Struktur

${ }^{8}$ Boas $(1911,30)$ spricht bei Sprachen, wo „the whole group of relations of the nouns of a sentence is expressed by the verb" von "pronominal cases“. Die Nomina (Subjekt, Objekte) würden „merely as appositions to a number of pronouns" erscheinen. Unter dem Stichwort „cross-reference“ erwähnt Bloomfield (1933, $193 \mathrm{f}$.), daß in vielen Sprachen „verb-forms include substitutive (pronominal) mention“ von verschiedenen (syntaktisch explizierten oder nicht explizierten) Konstituenten.

9 In der Sprachgermanistik schließt Erben $(1980,80)$ sich Weinrichs Kongruenzauffassung an. 
mit der konezntrischer Kreise um einen ins Wasser geworfenen Stein herum vergleichen: Sobald der Stein (das Verb) im Wasser ist (realisiert wird), erscheint eine Anzahl konzentrischer Kreise (Realisierungsschichten grammatischer Kategorien und lexikalischer Einheiten). Bedingung der Erscheinung des Kreises ${ }^{n}$, wobei $\mathrm{n}$ die in Schichtenzahl ausgedrückte Entfernung des Kreises vom Verb angeben soll, ist die des Kreises ${ }^{\mathrm{n}-1}$. Bereits hier erscheint also der entscheidende Gedanke vom Primat, d. h. von der Obligatheit, der Morphologie gegenüber syntaktisch-lexikalischen Aktantenrealisierungen, obwohl ja Weinrich unter ,Subjekt' weiterhin nur das syntaktische Subjekt versteht. ${ }^{10}$

\title{
1.3.3.
}

Exkurs: eine Gegenthese - John Lyons (1971 [Orig. 1968])

Den strukturellen Unterschieden zwischen Sprachen hinsichtlich der Subjektrealisierung versucht Lyons (1971, 284 f.) durch Einführung eines abstrakten, pronominalen Elements gerecht zu werden:

\begin{abstract}
„Nach traditioneller Auffassung wird die Person als Kategorie des Verbums betrachtet. Tatsächlich ist die Person in vielen Sprachen in der Flexionsform des Verbums oder der Verbalgruppe markiert. [...]. Im Lateinischen wird wie in vielen anderen Sprachen das Pronominalsubjekt normalerweise nicht angeführt: ego amo, tu amas u. s. w. würde nur dann gebraucht werden, wenn ego (〈ich $\rangle), t u(\langle\mathrm{du}\rangle)$ u. s. w. betont sind oder einer anderen ausdrücklich genannten oder vorausgesetzten Form gegenübergestellt werden. Aufgrund derartiger Sachverhalte wird oft die These vertreten, da $\beta$ die Selektion des Pronomens durch die Verbform bestimmt wird: daß also amo bei Betonung ego selegiert [...] u. s. w. Diese These ist jedoch falsch, da sie die Unterscheidung zwischen der 〈Oberflächenstruktur〉 und der ihr zugrundeliegenden 〈Tiefenstruktur〉 nicht berücksichtigt. Was die den Sätzen zugrundeliegende, semantisch interpretierbare Struktur betrifft, unterscheidet sich das Lateinische wenig vom Deutschen oder Englischen. In beiden Fällen müssen wir ein abstraktes 〈pronominales〉 Element (das nach Zahl und Person festgelegt ist) voraussetzen, welches Subjekt zum Verb ist und ausschlaggebend ist für die Regeln, dic für die phonologische Realisation des Verbums in der Oberfläche maßgeblich sind. Dieses 〈pronominale〉 Element wird im Lateinischen normalerweise nicht realisiert. Bei der Erzeugung von lateinischen Sätzen wird es getilgt, nachdem das Verb mit den Merkmalen von Person und Zahl ausgestattet worden ist, während es im Deutschen und Englischen gemäß den Regeln für die phonologische Realisierung als 〈Personalpronomen〉 [...]〈erzeugt〉 wird.“
\end{abstract}

Diese Auffassung ist von unserem Gesichtspunkt aus aufschlußreich, weil hier Selektion durch Verbmorphologie als eine einzelsprachlich-variierende, letztendlich also sekundäre Erscheinung einer universell-einheitlichen Potenz gegenübergestellt wird. Dabei erliegt man dem fundamentalen Irrtum, strukturelle Realisierungsbedingungen grammatischer Kategorien und deren grammatisch-kommunikative (d. h. grammatische und/oder kommunikative) Modifikationen (vgl. 1.3.5.3.) nicht auseinanderzuhalten bzw. erstere für die letzteren zu halten, um Platz für die „Tiefenstruktur“ zu schaffen. Die strukturellen

10 In seiner „Textgrammatik der französischen Sprache“ (1982) vertritt er seine frühere Expansionsthese nicht mehr. 
Realisierungsbedingungen sind von Sprache zu Sprache anders (vgl. unten 1.3.5. -1.5.), und sie stecken den Rahmen für die grammatisch-kommunikativen Modifikationen ab. Daß z. B. ich liebe im Gegensatz zu ego amo nur bei Betonung des Personalpronomens emphatisch ist (sein kann!), ist kein unbedeutender „Oberflächenunterschied“, sondern folgt aus einem wesentlichen strukturellen Unterschied zwischen Latein und Deutsch. Der Gedanke des „abstrakten pronominalen Elements“ (im übrigen auch: des leeren Subjekts im Rahmen des pro-drop-Parameters der GB, vgl. 1.4.4.) ist nur ein mehr oder weniger bewußter Versuch, demjenigen Strukturtyp universale Geltung zu verleihen, der die These von der grundsätzlichen Zweiteilung des Satzes in Subjekt und Prädikat zu bestätigen scheint (vgl. auch Anm. 22).

1.3.4. In seiner Reanalyse des Hockettschen Satzes She likes fresh milk verknüpft Jean Fourquet (1970, $20 \mathrm{ff}$.) Vorteile der Tesnièreschen Analyse mit denen der Weinrichschen. Gegen die von Hockett festgestellten unmittelbaren Konstituenten she, like-s und fresh milk hat er einzuwenden, ,,daß -s in like-s nicht dem Verb angehört, sondern dem Subjekt, es bringt eine Angabe über die Person, der die ,Liebe zur frischen Milch' eigen ist [...]. Dieselbe Mitteilung ist auch in she implizit, das noch eine Information (weibliches Wesen) bringt.“ (1970, 21) ${ }^{11}$ Überhaupt gilt:

„Das Verb ist eine Art Mikrokosmos, da hier in einem Segment der Kette neben dem Lexem ein Teil des Subjekts und die Anzeiger für Tempus und Modus (der Deckteil der verbalen Einheit) enthalten sind.“ (1970, 56)

Gleichwohl argumentiert Fourquet nicht nur für die integrative Sicht im Geiste Tesnières, ohne jedoch die Sonderstellung des Subjekts aufzugeben, sondern nimmt auch - wenn auch nur implizit und auch nicht mit dem glücklichsten Beispiel - für den Primat der Verbmorphologie Stellung (1970, $22 \mathrm{f}$.):

„Von der älteren Grammatik übernehmen auch entschiedene Neuerer den Begriff ,Kongruenz'. Es hieße z. B. von likes im Beispielsatz she likes fresh milk: das Verb nimmt die Form likes statt like, wenn das Subjekt ein Singular ist, und keiner der Teilnehmer der Mitteilung; d. h. likes wäre eine kon textbedingte Variante von like. Wir hätten demnach marchons als eine kontextbedingte Variante von marche [...] aufzufassen, die da aufträte, wo das Subjekt das Pronomen der 1. Pers. Plur. ist. Aber ein Telegramm arrivons demain ist für einen Franzosen eine sinnvolle

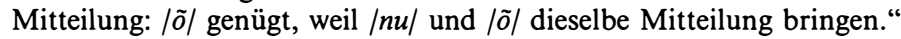

Hier wird also mit einer textsortenbedingten Verwendung für den Primat der Verbmorphologie eingetreten. Deutlicher vertritt er diese Ansicht, wenn er in griechisch trekhei, er läuft' die ohne syntaktische Expansion auftretende sog. spezifische Einheit (Glied) für 3. Pers. Sing. als das Subjekt ausweist (vgl. 1970, 56).

1 Fourquet lehnt sich hier an die Auffassung Martinets von den „signifiants discontinus“" an (vgl. Martinet (1980), 104). 
Fourquet weist auch darauf hin, daß durch seine Auffassung der Begriff der Kongruenz ,problematisch gemacht, wo nicht ganz beseitigt (wird).“(1970, 34)

1.3.5. Die integrative Sicht, die die Tesnière-Weinrich-Fourquet-Linie verkörpert, fordert einen geradezu heraus, nach einer Verbindung von Valenzansatz und Sprachtypologie zu suchen. Es war Fritz Pasierbsky (1981), der sich dieser Herausforderung angenommen hat.

1.3.5.1. Pasierbsky führt die Begriffe ,Makrovalenz' und ,Mikrovalenz' ein: Makrovalenz bezieht sich ,,auf die morphologisch-syntaktischen Beziehungen zwischen (prädikativem) Verb und anderen Redeteilen“ (1981, 162), es geht hier also um die traditionell als syntaktische Valenz bezeichnete Fähigkeit. Mikrovalenz ist „die Eigenschaft eines Morphems in der Funktion eines Valenzträgers, verschiedene Aktanten (eventuell auch Zirkumstanten, z. B. adverbiale Zeitbestimmungen), die makrovalente Leerstellen im Satzbauplan besetzen (bzw. als freie Angaben benutzt werden), in der morphologischen Struktur des Verbs zu repräsentieren. Für einen interlingualen Vergleich ist es [...] von Interesse, [...], in welcher typischen Art und Weise die morphologische Struktur des Verbs den Satz in konzentrierter Form zum Ausdruck bringt.“ (ebd., 163) ${ }^{12}$ Das (in der Art Fourquets gezeichnete) Stemma von Damals kannten wir diesen Menschen nicht sieht demnach wie folgt aus (ebd., 165): ${ }^{13}$

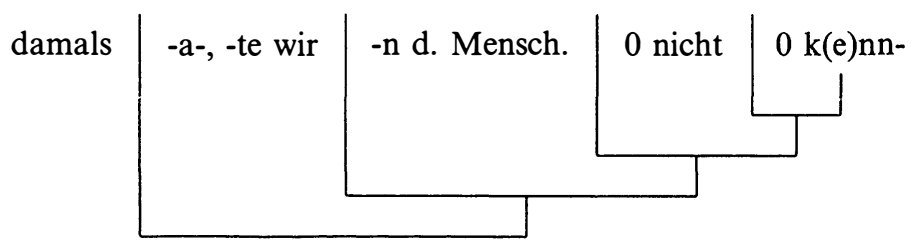

Es ist ersichtlich, daß damals und wir über mikrovalenzielle Repräsentationen im verbalen Valenzträger verfügen $(-a-$, $-t e$ bzw. $-n)$, während die Akkusativergänzung und die Negation nur makrovalenziell in Erscheinung treten (diesen Menschen und nicht). Das Stemma macht auch deutlich, daß Mikrovalenz ein wichtiges Argument liefert, „das finite Verb als strukturelles Satzzentrum zu behandeln." (ebd., 163)

12 Pasierbsky lehnt sich an Fourquets Mikrokosmos-Gedanken an. Repräsentation ,ist die Realisierung eines oder mehrerer Aktanten der makrovalenten Ebene in der mikrovalenten morphologischen Struktur des verbalen Valenzträgers." $(1981,167)$ Diese Bestimmung läßt allerdings offen, ob die Repräsentation eine Form der Selektion ist oder ob es sich um eine andere, nicht näher bestimmte Art von Relation handelt.

13 Links von einem terminalen Knoten erscheinen die makrovalenten Aktanten und Zirkumstanten, rechts davon ihre mikrovalenten Repräsentanten. „-a-“ ist der präteritale Stammvokal von kenn-. 
Durch die Untersuchung des Zusammenspiels der Makro- und der Mikrovalenz in verschiedenen Sprachen kommt man zu Repräsentationstypen, denen die Sprachen der Welt zugeordnet werden können. ${ }^{14}$

Pasierbsky thematisiert auch die Frage der Repräsentationsrichtung (der strukturellen Aktantenrealisierungsrichtung). Er versucht $\mathrm{zu}$ begründen, warum er im Gegensatz zu Erbens und Weinrichs zentrifugaler Repräsentationsauffassung für die zentripetale eintritt, d. h., warum er am Primat der Syntax gegenüber der Verbmorphologie festhält (1981, 168 ff.):

(a) Da er in Anlehnung an die bereits zitierten Ausführungen von Lyons ,Numerus' und ,Person' als nominale Kategorien (der Tiefenstruktur) interpretiert, erblickt er in der morphologischen Struktur des Verbs (u. a.) die formale Kennzeichnung nominaler Aktanten. ${ }^{15}$

(b) Sprachgeschichtlich sind Personalformen der indoeuropäischen Verben, Kongruenz (und überhaupt die Flexionen) als Ergebnis von Pronominalisierungsprozessen zu erklären: ursprünglich selbständige Pronomina verschmolzen mit dem Verbum.

1.3.5.2. Zu (a): Nach Pasierbsky müsse also der Status der Kategorien ,Numerus' und 'Person' (nominal oder verbal) die (Aktanten)Repräsentationsrichtung festlegen: Da eine nominale Kategorie per definitionem primär am Nomen (Makroaktanten) realisiert wird, ist ihre Realisierung am Verb per definitionem sekundär. Demnach sei die mittels der Kongruenz ausgedrückte Repräsentationsrichtung zentripetal.

Das Problem dieser Auffassung ist ihre Rigidität. Mit der Entscheidung, ,Numerus' und ,Person' seien nominal (oder eben verbal), macht man den Status dieser Kategorien, ausgerechnet in einer strukturell-typologischen Untersuchung, von den strukturellen Realisierungseigenschaften in den Einzelsprachen unabhängig. Daraus folgt, daß die Festlegung der Aktantenrepräsentationsrichtung ebenfalls einzelsprachunabhängig bzw. notwendigerweise an den Status von ,Numerus' und/oder ,Person' gebunden ist.

Ich bin der Meinung, da $\beta$ die u. a. von Pasierbsky beschriebenen typologischen Unterschiede eines flexibleren methodischen Rahmens bedürfen. Eine solide Basis für einen solchen Rahmen könnte zum Teil aus Eisenbergs Feststellungen abgeleitet werden. In seinem Modell setzen nämlich syntagmatische Relationen voraus, daß jede an einer Relation teilhabende Konstituente über eine entsprechende (Konstituenten)Kategorie verfügt, da eine Konstituente nur kraft einer Kategorie eine Relation eingehen kann. In diesem methodischen Kontext hätte folglich etwa die Aussage, ,Numerus' sei eine nominale (Konstituenten)Kategorie oder ,Numerus' sei eine verbale (Konstituenten)Kategorie, keinen Sinn. Syntagmatische Relationen sind Relationen, die sich ein-

14 Pasierbskys Typologie (1981, $171 \mathrm{ff}$.) geht bis zu drei Aktanten. Vgl. auch die 60 Sprachen umfassende Tabelle in Nichols (1986), $68 \mathrm{f}$.

15 Bei der Kongruenz handelt es sich ,um die Realisierung nominaler Kategorien am Verb“ [...]. (1981, 169) 
stellen, wenn beide - nominale und verbale - Konstituentenkategorien realisiert werden können oder müssen. Dabei bleibt die Frage vorläufig (vgl. 1.4.2.2. - 1.4.2.4.) offen, ob diese Realisierung an gleichbleibende Konstituenten gebunden ist.

Mit der Metapher sich einstellen soll nicht der Anschein erweckt werden, als ob Makro- und Mikroebene funktional unabhängig sind. Der große Vorteil von Pasierbskys Lösung, der in der funktionalen Zuordnung der beiden Ebenen (vgl. auch das obige Stemma) liegt, darf nicht aufgegeben werden. Deshalb möchte ich dafür plädieren, daß man zwischen Konstituentenkategorien (im Sinne Eisenbergs), die per definitionem immer an einer Konstituente realisiert werden, und ihnen zugrundeliegenden (einzelsprachlichen) funktionalen Kategorien, die an einer oder mehreren Konstituenten realisiert werden können, unterscheidet. Der Status einer funktionalen Kategorie soll dann in Abhängigkeit von den Realisierungseigenschaften ihrer Konstituentenkategorien bestimmt werden.

Daß eine Trennung der Kategorienrealisierungen möglich und in typologisch orientierten Untersuchungen auch notwendig ist, dafür gibt es empirische Hinweise. ${ }^{16}$ Bei Sprachen wie Deutsch, in denen das Verb sowohl über Paradigmen- wie auch über Einheitenkategorien verfügt, ist dieser Umstand selbst ein empirischer Hinweis, denn während sich die einzelnen Einheitenkategorien bei den verschiedenen Formen desselben Verbs abwechseln, bleibt die Paradigmenkategorie per definitionem konstant. Und methodologisch muß die Trennung schon deshalb zulässig sein, weil die einzelnen Kategorien sauber unterscheidbare syntagmatische Relationen begründen (1.2.).

Die weitaus schwierigere Frage ist allerdings die der Methode. Können die Realisierungseigenschaften der einzelnen funktionalen Kategorien überhaupt getrennt werden, wo doch diese Kategorien offensichtlich intensiv interagieren und oft auch amalgamiert realisiert werden (vgl. auch Bossong (1980), 9). Wie kommt man beispielsweise an Kategorien wie z. B. dt. ,Nominativ', ,Person` und ,Numerus' einzeln heran?

Unter den Kategorien ,Person' und ,Numerus' gebührt die primäre Bedeutung aus funktionaler Sicht gewiß den Personenkategorien, da sie die Äußerung in der Sprechsituation, im Kommunikationsdreieck (vgl. Weinrich (1967), 111) lokalisieren. ${ }^{17} \mathrm{Zu}$ erwarten ist daher eine Arbeitsteilung zwischen Personen- und Kasuskategorien in dem Sinne, daß

„person and case are two central relational grammatical categories, which achieve the integration of the sentence and its reference to the speech situation: case relates the arguments to the relational expression and ultimately to the expressed event;

16 Vgl. auch Andrews (1985), $75 \mathrm{f}$. Daß reiche Flexionsmorphologie und Zulässigkeit des pro in keiner direkten Beziehung stehen, zeigen auch GB-orientierte Arbeiten wie z. B. Huang (1984); Jaeggli/Safir (1989), 26 ff. und Franks (1990). Vgl. auch 1.4.3.

17 ,Numerus', der für unsere Betrachtungen weniger wichtig ist, wird deshalb im folgenden vernachlässigt. 
person relates the relational expression to the arguments and ultimately to the participants of the speech situation." (Lehmann (1982), 217) ${ }^{18}$

Wie sind nun die (komplexen) ${ }^{19}$ funktionalen Kategorien ,Person' und ,Nominativ‘ im Deutschen zu bewerten? Als Beispiel soll du gehst fungieren, wobei auf die „starke Annahme“ (vgl. 0.2.) eines paradigmatischen Erklärungsmusters (Typ: „reiches Flexionsparadigma“) von vornherein verzichtet wird, da man sonst unabhängige Kriterien heranziehen können müßte, um zu zeigen, wie Paradigmen genau „funktionieren“:

Beide Konstituenten - $d u$ wie gehst - sind personal und kasuell eindeutig identifizierbar. Diese Symmetrie ist jedoch trügerisch, wie der Vergleich der Formen $\left(^{*}\right)$ gehst und *du geh nahelegt, denn nur gehst, nicht aber du geh, ist als Ellipse möglich (vgl. 5.). Wie ist das nun mit der Realisierung von ,Nominativ' und ,Person' in Verbindung zu bringen?

Die Antwort ist naheliegend, wenn wir auf die Eisenbergschen Relationen rekurrieren. Da ,Nominativ“ im Deutschen eine Rektionsbeziehung mit verbaler Paradigmenkategorie eingeht, da also die Quelle dieser Relation die Verbform ist, muß die funktionale Kategorie als ,verbaler Nominativ' bestimmt werden.

Umgekehrt liefert die Kongruenzbeziehung zwischen gehst und $d u$ zwar weder für den nominalen noch für den verbalen Status von ,2. Person“ ein Argument, es bleibt jedoch für die Erklärung der Ellipse gehst nur die Möglichkeit der Annahme der Kategorie ,nominale 2. Person'. Denn diese Ellipse kann nur aus bestimmten grammatisch-kommunikativen Konstellationen hervorgehen, aus Konstellationen also, die Druck auf die Nichtrealisierung von $d u$ ausüben. Und daß es dieses Druckes überhaupt bedarf, heißt, daß es einen anderen, permanenten Druck - eben den normalen Druck auf die nominale Realisierung von $d u$ - gibt. Da dieser Druck angesichts des verbalen Status der Nominativkategorie nicht - oder nicht allein - von dieser aus-

${ }^{18}$ Genau an diesem Punkt zeigt sich die (nicht zwingende, aber naheliegende) Theorieabhängigkeit der Entscheidung ,Zweiteilung oder Verbzentriertheit des Satzes' sehr deutlich. Denn während für eine Konstituentengrammatik eher ,Person' und ,Numerus“ als prominente Kategorien in Frage kommen, da sie in der Regel „sichtbare“ syntagmatische Relationen zwischen Subjekts-NP und VP herstellen, sind für eine Abhängigkeitsgrammatik eher die Kasuskategorien von grundlegender Bedeutung, da diese die wohl auffälligsten Vertreter der Determinationsfunktion (im Sinne Hjelmslevs) sind. Eine restriktive Phrasenstrukturbeschreibung, wie sie für die kategoriale Komponente der GB verwendet wird, bedient sich daher eines eigenen Knotens für die Kongruenzmerkmale von Subjekt und Prädikat (INFL, vgl. auch 1.4.4.). Demgegenüber wird ein vergleichbarer(!) Knoten nicht einmal in dem Dependenzmodell von Eroms (1985) vorgeschlagen, obwohl dort die Personen- und Numeruskongruenz als ein wichtiges Argument angesehen wird, das syntaktische Subjekt direkt an das jeweilige Finitum - auch wenn es ein Hilfsverb ist - anzubinden.

19 „Komplex“ wird eine beliebige Kategorie genannt, wenn sie eine methodisch begründete, abstrakte „Zusammenlegung“" von (funktionalen oder Konstituenten-)-Kategorien darstellt. Es handelt sich also um Hilfskategorien, die u. U. eine vereinfachte Darstellung ermöglichen. 
gehen kann, ist die funktionale Personenkategorie als ,nominale 2. Person“ anzusetzen.

1.3.5.3. Das valenzgrammatisch Interessante an Kategorien wie ,verbaler Nominativ' und ,nominale Person' ist jedoch nicht ihr genauer Status, sondern $\mathrm{da}$ für die Beschreibung ihrer Interaktion das von Pasierbsky eingeführte Ebenen-Konzept der strukturellen Valenzrealisierung einen geeigneten Rahmen bietet. Um beim Beispiel $d u$ gehst zu bleiben:

Da geh- die Realisierung des Verblexems darstellt, kommen nur die Makroebene $d u$ und die Mikroebene -st als potentielle n o minale materielle Träger der (materiell amalgamiert erscheinenden) funktionalen Kategorien ,Nominativ', ,Singular' und ,2. Person' in Frage. Gleichzeitig sind sie potentielle materielle Träger der in der deutschen Valenzforschung angesetzten Kategorie ,Nominativergänzung'20 (syntaktisches „Subjekt“), woraus man schließen muß, daß diese valenzgrammatische Kategorie des Deutschen auf der nominalen Realisierung der funktionalen Kategorie ,Nominativ' und/oder der funktionalen Personen- und Numeruskategorien basiert. Dabei dürfte der Unterschied zwischen ,Valenzrealisierung' und ,Summe der Kategorienrealisierungen“ gerade darin bestehen, daß eine Valenzrealisierung keine mechanische Summe der Kategorienrealisierungen darstellt.

Um diesen Unterschied einzufangen, soll der Begriff ,strukturelles Deiktikon/Anaphorikon" - eine Unterscheidung zwischen ,Deiktikon“ und ,Anaphorikon“ ist für die Zwecke der vorliegenden Arbeit nicht notwendig, vgl. z. B. Bossong (1980), 9 ff. - eingeführt werden. Darunter verstehe ich in Anlehnung an die Zweifelderlehre Bühlers (1934) ein Element des Zeigfeldes, das seine hinweisende Funktion nicht als Aufhebungs-, Reduktions- oder Expansionsform unter besonderen grammatischen und/oder kommunikativen Bedingungen ausübt. Diese Feststellung impliziert die Unterscheidung von Äußerungsformen, die $1: 1$-Realisierungen von Grundstrukturen der betreffenden Sprache sind, von denen, die Realisierungen entweder von grammatisch normgemäßen oder pragmatischen (varietätenbezogenen, textsortenspezifischen) Modifikationen dieser Grundstrukturen darstellen (die Begriffe ,System, Norm und Rede' werden in der ganzen Arbeit im Sinne Coserius verwendet, vgl. z. B. ders., (1988), 266 ff.). Der Terminus für pragmatische Modifikationen ist ,Aufhebung'. Die Termini für grammatische Modifikationen sind in Abhängigkeit davon, ob im Vergleich zu realisierten Grundstrukturen ein Zuwachs

${ }^{20}$ Die oft synonym gebrauchten Begriffe ,Nominativergänzung' und ,Erstaktant' sollen hier strikt getrennt werden. ,Nominativergänzung' als einzelsprachliche Kategorie ist als eine einzelsprachliche Erscheinungsform des allgemein-dependenziellen Begriffs ,Erstaktant' im Sinne Tesnières aufzufassen. Dabei soll jedoch im Unterschied zu Tesnière an letzteren kein Bezeichnungstyp ,Agens' herangetragen werden (Bossong (1980), 5). Genausowenig wird durch „Erst-“ irgendeine Rangfolge ausgedrückt. Mehr zur ,Nominativergänzung' in 1.4. 
an oder eine Abnahme von Elementen verzeichnet werden kann, ,Expansion“ bzw. ,Reduktion' (vgl. ausführlicher 1.3.7., 1.4. und 5.).

In diesem Sinne sind die indikativischen Verbflexive des Deutschen - im Gegensatz zu denen vieler anderer Sprachen wie des Italienischen, Spanischen, Serbokroatischen(!) oder Polnischen - keine strukturellen Deiktika/Anaphorika, weshalb die ausschließlich mikrovalenzielle Besetzung der Nominativstelle im (strukturellen) Normalfall unangemessen ist (genauer und ausführlicher in 1.4.2.). Dabei ist die Realisierung der mikrovalenziellen Ebene strikt obligatorisch, weshalb eine mikrovalenzielle Nichtbesetzung nicht einmal in grammatisch-kommunikativen Modifikationen vorkommen kann (vgl. 2.2. und 5.). Die Obligatheit bedeutet aber keinesfalls, daß man hier von "Redundanz" sprechen müßte. (Für zwei mögliche Erklärungsmuster der sog. morphologischen Redundanz vgl. Heger (1991) und Ágel (1991)).

$\mathrm{Da} B$ nun eine nur makrovalenzielle Erstaktantenrealisierung im Deutschen bzw. in allen Sprachen mit mikrovalenziellen Repräsentationen nicht möglich ist, daß umgekehrt alle Sprachen mit mikrovalenziellen Repräsentationen, wobei die Verbflexive strukturell deiktisch/anaphorisch sein müssen, eine nur mikrovalenzielle Repräsentation möglich machen, spricht für den Primat der morphologischen Valenzrealisierungsebene. ${ }^{21} \mathrm{Ob}$ dabei der Begriff der Repräsentation beizubehalten ist, soll vorläufig (bis 1.4.2.4.) dahingestellt bleiben.

1.3.5.4. Zu (b): Im Lichte des Gesagten wiegt Pasierbskys zweites Argument für den Primat der Syntax, sprachgeschichtlich seine Personalformen der indoeuropäischen Verben, die Kongruenz und überhaupt die Flexionen als Ergebnis von Pronominalisierungsprozessen zu erklären, zwar weiterhin schwer, man kann ihm aber doch einiges entgegensetzen:

(b 1) Die Trennung der strukturellen Realisierungsbedingungen der Personen(- und Numerus)kategorien von denen der Kasuskategorien macht die Frage möglich, ob die genannten Pronominalisierungsprozesse primär nicht die strukturellen Realisierungsbedingungen der Personen(- und Numerus)kategorien betrafen und die der Kasuskategorien, materiell angebunden, nur „mitgeschleppt“ wurden (vgl. auch 2.2.). Denn sicherlich ist die wichtigste Voraussetzung dafür, daß die Verbform zu einem „Mikrokosmos“ werden kann, ihre Einbindbarkeit in das Kommunikationsdreieck. Freilich kann diese Argumentation vorrangig nur für den Bereich des Erstaktanten gelten, im Bereich des Zweitaktanten scheinen die von Christian Lehmann (1982, 234 f.) aufgestellten Typen von Kongruenzbeziehungen in natürlichen Sprachen für

21 Bei Sprachen ohne mikrovalenzielle Repräsentation ist er eine Frage der Festlegung bzw. der Zielsetzung der Untersuchung, ob man vom Primat der (0-)Morphologie spricht. Vergleicht man Valenzrealisierungsebenen in verschiedenen Sprachen, ist jedoch der Begriff des 0-Mikroaktanten kaum zu vermeiden, da die meisten Sprachen über (eine unterschiedliche Anzahl von) sowohl Mikroebenen wie auch morphologisch markierte(n) Makroebenen verfügen. Vgl. 1.3.6. und 1.3.7. 
eine sukzessive kasuelle Klitisierung des Personalpronomens zu sprechen (vgl. auch Givon (1984), 353 ff. und den Überblick in Bynon (1990)). Voraussetzung ist allerdings, daß man diese ,synchronen“ Typen oder einen Teil von ihnen als „diachrone“ Stufen derselben historischen Sprache anerkennt und/oder identifizieren kann (vgl. Bynon (1990), $100 \mathrm{ff}$.).

(b 2) Sprachgeschichtlich bekannt ist nicht nur der Weg von der Syntax zur Morphologie, sondern auch der umgekehrte. Die Veränderungen in der Flexionsmorphologie, die Synkretismen bewirken (besser: tragen dazu bei), daß ein „Mikrokosmos“, egal ob nominaler oder verbaler, expandiert wird. Dies ist der Weg, den man gewöhnlich als Analytisierung des Sprachbaus beschreibt. Dabei ist die Frage kaum (pauschal, einzelsprachunabhängig) zu beantworten, ob für derartige Veränderungen in erster Linie die (teils) zerstörten strukturellen Realisierungsbedingungen der Personen(- und Numerus)kategorien oder der Kasuskategorien verantwortlich zu machen sind, d. h., in primär welchem Bereich die Sprachteilhaber eine Kommunikationsstörung (unbewußt) beheben wollten.

1.3.6. In einer umfassenden typologischen Untersuchung befaßt Johanna Nichols (1986) sich mit den möglichen Kombinationen und Typen von morphologischen Markierungen verschiedener syntaktischer Relationen in verschiedenen Sprachen. Dabei stützt sie sich auf zwei grundlegende Konzepte: „headedness“ (aus: „head“ „Kopf”) und (morphological) „marking“. Mit ihrer Hilfe untersucht sie syntaktische Konstruktionen auf drei Ebenen (phrase, clause, sentence) daraufhin, ob die innerhalb der Konstruktionen bestehenden syntaktischen Relationen am Kopf oder am „dependent“ (etwa: ,Komplement”) bzw. an beiden oder keinem von beiden morphologisch markiert werden. Nach den Markierungspräferenzen lassen sich dann die Sprachen zwischen den Polen von „typical head-marking language“ und „typical dependent-marking language" lokalisieren.

Unter den von Nichols untersuchten syntaktischen Ebenen befindet sich auch der Einfachsatz („clause“). Seine Struktur wird u. a. daraufhin geprüft, ob Kasus(form)informationen nur am Prädikatsverb (,head-marked type“), nur an den restlichen Phrasen (,dependent-marked type“), seien diese Ergänzungen oder Angaben, oder an beiden bzw. keinem von beiden morphologisch erscheinen.

Der entscheidende Fortschritt in Nichols' Arbeit gegenüber dem Mikrokosmos-Gedanken von Fourquet und den zwei strukturellen Valenzebenen Pasierbskys (bzw. den entsprechenden Bemühungen der GB im Zusammenhang der Kategorien ,pro' und ,INFL') besteht darin, daß die Relation von „head“ und „dependent" in Sprachen mit „head-marked-clauses“ als eine notwendigerweise andere angesehen wird wie in denen mit „,dependent-markedclauses“ (1986, $107 \mathrm{ff}$.$) , denn: „In head-marked grammatical relations, the$ dependent is usually an optional element of the constituent." (ebd., 107) Die Mikrokosmos-Metapher Fourquets trifft also den Kern der Sache genauge- 
nommen nur beim „head-marking“: ,[...] in languages with consistently headmarked clauses, the verb itself normally constitutes a complete sentence; full NP's are included only for emphasis, focus, disambiguation etc. [...] The dependent in a head-marked constituent stands in a roughly appositive relation to the head (or, more precisely, to the coreferential marker on the head) [...]." (ebd., 107) Im übrigen steckt hierin eine mehr oder weniger explizite Kritik an dem Verfahren der GB, den dependent-marking-Typus zum Archetyp universalgrammatischer Untersuchungen gemacht zu haben. ${ }^{22}$

1.3.7. Es geht u. a. aus der bisher zitierten Literatur hervor, daß es viele Sprachen mit strukturell deiktisch-anaphorischen Verbflexiven (in der GB: „pro-drop-Sprachen“ oder „Null-Subjekt-Sprachen“) gibt. Werden die Verbvalenzen einer solchen Sprache mit denen einer beliebigen anderen kontrastiert, könnte man erwarten, daß die Übertragbarkeit des für das Deutsche ausgearbeiteten syntaktischen Valenzmodells überprüft und diskutiert wird. Obwohl derartige Erwartungen nicht nur methodologisch begründbar erscheinen, sondern auch durch Interferenzuntersuchungen nahegelegt werden (vgl. beispielsweise Ulbricht (1987)), ist dies jedoch nicht oder nur ansatzweise der Fall.

Eine konsequente Absage an die unkontrollierte Übertragung des syntaktischen Valenzmodells erteilt erst Sarolta László. Ihre Analysen führen sie bereits 1978 dazu, „die Anwendbarkeit desselben Valenzbegriffs auf die zwei Sprachen [Deutsch und Ungarisch - V. Á.] in Frage (zu) stellen. “ $(1978,164)$ Eine elaborierte Untersuchung der minimalen Verbstrukturen in ungarischdeutscher Relation legt sie dann 1988 vor.

László (1988) nimmt in Anlehnung an Pasierbsky zwei Realisierungsebenen der Valenz, die Mikroebene (die der sog. morphologischen Aktanten) und die Makroebene (die der syntaktischen Aktanten) an. (Für „das Ungarische ,morphologische Aktanten' anzunehmen, d. h. die deutschen Personalpronomen und die ungarische Flexion als gleichwertige Elemente zu betrachten“, erwog sie schon in László (1978), 165.) Die Aktanten, die auf beiden Ebenen realisiert werden können (im Deutschen nur die Nominativergänzung, im Ungarischen Nominativ- und Akkusativergänzung), heißen Zwei-Ebenen-Aktanten. Im Gegensatz zu Pasierbsky geht aber László vom Primat der Verbmorphologie aus:

„Der obligatorische Charakter der Morphologie, der Umstand, daß die ZweiEbenen-Aktanten morphologisch immer bezeichnet werden müssen, während sie

22 Auch van Valin (1985) weist nach, daß die GB als eine „English-based theory of grammatical structure“ (ebd., 363) unfähig ist, Sprachen mit „head-marking nature“ wie z. B. das Lakota adäquat zu beschreiben. Die Beschreibungsinadäquatheit der GB resultiere daraus, daß diese Theorie insgesamt eine „dependent-marking orientation" aufweise (ebd., 375). Derselben Ansicht ist Comrie (1989, 227 f.). Eine Zusammenfassung GB-interner Ansichten über den Zusammenhang von „pro-drop“, Verbmorphologie und Kongruenz geben z. B. Hermon/Yoon (1989), Müller/Rohrbacher (1989) und Jaeggli/Safir (1989), 9 ff. Vgl. auch 1.4.4. 
syntaktisch unbezeichnet bleiben können, spricht dafür, daß man den Satz von der Verbform herleitet. In bezug auf das Ungarische kann man dabei davon ausgehen, $\mathrm{da} B$ in einer ungarischen Verbform ein oder zwei Aktanten des Verbs in Mikroform realisiert sind. Die Mikroformen können als Deiktika/Anaphern selbständig funktionieren, sie sind also potentielle Zeigfeld-Aktanten und die Verbformen selbst oft ein potentieller Zeigfeld-Satz. Eine syntaktische Explizierung der Mikroformen wird nötig, wenn ihre Information nicht ausreicht. Grundsätzlich ist dies bei Topikalisierung im Zeigfeld und im Symbolfeld der Fall.“" 23

Die strukturell-typologischen Unterschiede zwischen Sprachen sind an Gegenüberstellungen von Vorkommensrelationen zwischen den Einheiten der beiden Ebenen abzulesen (1., 2. usw. = mögliche Strukturkorrespondenzen, $\mathrm{a}=$ Mikroform, $\mathrm{A}=$ Makroform, $0=$ Null-Realisierung). Als Beispiele mögen ung. eszik und dt. essen dienen: ${ }^{24}$

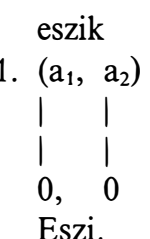

2. $\left(a_{1}, a_{2}\right)$<smiles>IO[AlH]I</smiles>

$\mathrm{A}_{1}, 0$

$\mathrm{Az}$ anya eszi.

3. $\left(a_{1}, a_{2}\right)$<smiles>I[V]I</smiles>

Eszi a húst.

4. $\left(a_{1}, a_{2}\right)$<smiles>I[I-]I</smiles>

$\mathrm{A}_{1}, \mathrm{~A}_{2}$

$\mathrm{Az}$ anya eszi a húst.

5. $\left(a_{1}, 0\right)$<smiles>I[V]I</smiles>

Mindent eszik.

\section{essen}

$\left(a_{1}, 0\right)$

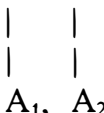

Sie ißt es.

Die Mutter ißt es.

Sie ißt das Fleisch.

Die Mutter ißt das Fleisch.

Sie ißt alles.

${ }^{23}$ László (1988), 227 f. Die morphologische Bezeichnung kann auch durch ein NullMorphem repräsentiert sein.

${ }^{24}$ eszik ist entsprechend der ung. Lemmatisierungskonvention Sing. 3. Pers. 1-4. wird es objektiv (definit), 5-6 subjektiv (indefinit) konjugiert. (Die Verben des Ungarischen werden mit definitem Zweitaktanten objektiv, mit indefinitem subjektiv konjugiert.) 
6. $\left(\mathrm{a}_{1}, 0\right)$

$\begin{array}{ll}\mid & \mid \\ A_{1}, & A_{2}\end{array}$

Anya mindent eszik. Die Mutter ißt alles.

Es ist ersichtlich, daß die definite Minimalstruktur (1.) des Ungarischen in jeder mathematisch möglichen Kombination expliziert werden kann $(2-4$.), während die deutschen Entsprechungen immer die gleiche, syntaktisch voll explizierte Struktur haben müssen. Auch gibt es im Ungarischen eine indefinite Minimalstruktur (5.) mit der dazugehörigen Explizierung (6.), denen auf deutscher Seite weiterhin die gleiche, syntaktisch voll explizierte Struktur entspricht. M. a. W., die nominativischen und akkusativischen syntaktischen Verweisformen sind im deutschen Indikativsatz bereits strukturell besetzt, während sie im Ungarischen zusätzliche grammatisch-kommunikative Aufgaben (Emphase, Kontrastierung) übernehmen können, ohne daß die Pronomina besonders akzentuiert werden müßten: Das morphologisch zu realisierende, konjugationsparadigmatische „Minimalpaar“ (objektiv vs. subjektiv) des Ungarischen kompliziert die Strukturverhältnisse, schafft aber Freiräume auf syntaktischer Ebene; die denkbar einfachste Strukturgrundlage des Deutschen sichert sich diese Schlichtheit durch die strukturelle Bindung syntaktischer Potenzen eine alte Erkenntnis, die durch Lászlós Strukturkorrespondenzen transparent gemacht wird.

Der aufmerksame Leser kann unter den obigen Strukturkorrespondenzen zwei der mathematisch möglichen Kombinationen nicht entdecken:

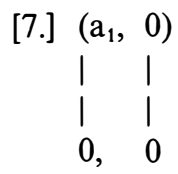

Eszik.

[8.] $\left(\mathrm{a}_{1}, 0\right)$

$\mathrm{A}_{1}, 0$

Anya eszik.

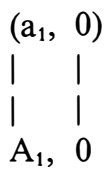

Sie ißt.

Daß László diese beiden Gegenüberstellungen nicht als Sturkturkorrespondenzen ansieht und daß sie als solche auch nicht angesehen werden können, folgt aus der Auffassung, die der Morphologie den Primat zuerkennt. Der große methodische Vorteil gegenüber der „syntaktizistischen“ Auffassung besteht u. a. darin, daß man sich nicht auf den schwankenden Boden der Ellipsenforschung begeben und dementsprechend auch nicht die Frage stellen muß, ob die Sätze in [7.] und [8.] Realisierungen von Strukturkorrespondenzen oder Realisierungen von reduzierten Strukturkorrespondenzen, d. h. indefini- 
ten Auslassungen, sind. ${ }^{25}$ Ohne das Gebiet der Ellipsenforschung betreten zu müssen, gewinnt man hier vielmehr eine sichere methodische Grundlage von der Verbstruktur her, die hinsichtlich der Elliptizität der Sätze in [7.] und [8.] nur eine Entscheidung zuläßt: Da das (in den Strukturkorrespondenzen Lászlós implizierte) strukturelle Kriterium der indefiniten Auslassung die makrovalenzielle Nichtrealisierung eines mikrovalenziellen 0-A ktanten ist, stellen alle Sätze in [7.] und [8.] indefinite Auslassungen (des Zweitaktanten) dar. Dieses Kriterium sagt übrigens korrekt voraus, daß eine indefinite Auslassung der Nominativergänzung im Deutschen nicht möglich ist (mehr zum Ellipsenproblem in 5.).

Auffallend ist an den Strukturkorrespondenzen Lászlós - aber auch sonst an den in der Literatur analysierten Verbbeispielen -, daß sie im Indikativ stehen. Offensichtlich sind wir hier Zeugen eines stillschweigenden Konsenses über den Primat, d. h. genauer: die strukturelle Ausschließlichkeit, des Indikativs gegenüber allen anderen möglichen Modi. Ich sehe jedoch keinen Grund, etwa den deutschen oder den ungarischen Imperativ als eine grammatische Reduktionsform des Indikativs anzusehen, ganz im Gegenteil: Eine solche Lösung führt nämlich dazu, daß die emphatische Setzung des Personalpronomens im Imperativ (Mach du es!) als eine Expansion einer Reduktion eingestuft werden muß - ein Status, dessen Erklärungsadäquatheit generell zu bezweifeln ist. Deshalb sollen die obigen Korrespondenzen durch drei weitere mit imperativischen Verbformen ergänzt werden:

9. $\left(a_{1}, a_{2}\right)$

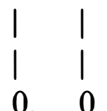

$0, \quad 0$

Edd!

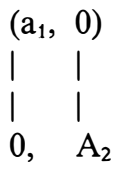

IB es.

10. $\left(a_{1}, a_{2}\right)$

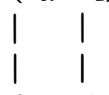

$0, \quad \mathrm{~A}_{2}$

Edd a húst!

IB das Fleisch!

11. $\left(a_{1}, 0\right)$

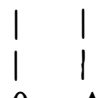

$0, \quad \mathrm{~A}_{2}$

Egyél mindent! I $\quad$ I alles!

Hier wiederholt sich haargenau die Konstellation des Indikativs: alle möglichen Strukturkombinationen auf ungarischer Seite, die gleiche Struktur

${ }^{25}$ Vom konkreten Sprechen her gesehen (vgl. Coseriu (1988)) sind Realisierungen von Grundstrukturen mit Expansions-, Reduktions- und Aufhebungsformen funktional gleichwertig. 
im Deutschen. Der Unterschied besteht darin, daß, während das Ungarische aus „mathematischen“ Gründen keine neue Strukturkombination mehr aufweisen kann, zu der deutschen Indikativstruktur eine neue Struktur im Imperativ hinzugekommen ist. Dies ist, was die Grammatiken des Deutschen traditionell mit einem Hinweis auf eine „Valenzreduzierung“ im Imperativ erledigen. M.E. ist jedoch der Fall anders zu sehen, denn der deutsche Imperativ drückt die Nominativergänzung strukturell deiktisch/anaphorisch genauso gut aus wie der Indikativ. M. a.W., I $\beta$ ! ist nicht weniger „subjekthaltig“ als $D u$ i $\beta t$, nur da $\beta$ die Nominativergänzung im Imperativ makrovalenziell nicht realisiert wird. Diese Auffassung impliziert, daß ein rein syntaktisches Valenzmodell nicht einmal für die Beschreibung des Deutschen ausreicht, denn auch das Deutsche verfügt über strukturell deiktisch-anaphorische Verbflexive, nämlich im Imperativ. Daß das syntaktische Valenzmodell schon immer als für deutsche Verhältnisse besonders geeignet empfunden wurde, ist demnach gewissermaßen dem Umstand zu verdanken, daß der Imperativ schon immer als abgeleiteter Modus angesehen wurde. ${ }^{26}$

\subsection{Zum Verhältnis der Ebenen im Zwei-Ebenen-Modell}

Der womöglich heikleste Punkt im Zwei-Ebenen-Modell ist das Verhältnis der beiden Ebenen zueinander. Denn einerseits schafft die postulierte Obligatheit der mikrovalenziellen Ebene eine sichere Grundlage für die Erfassung der valenziellen Vorkomensrelationen in den verschiedenen Sprachen, andererseits muß man über diese hinausgehen, indem man sich dem typologisch asymmetrischen, inhaltlichen Verhältnis der Ebenen zuwendet. Dabei geht es offensichtlich um das Problem, das als „die Verteilung der deiktisch-anaphorischen Potenz auf die Ebenen und deren modellinterne Bezugspunkte" umschrieben werden könnte. Als modellinterne Bezugspunkte der genannten Potenz kommen die Kasuskategorien und verschiedene Modifikationen (zum Begriff vgl. 1.3.5.3.) in Frage. Dies folgt sowohl aus unserem Plädoyer für eine Theorie des Sprechens im Geiste von Coseriu (1988) als auch aus der folgenden Valenzdefinition:

Unter Valenz soll die Potenz eines verbal oder nominal zu realisierenden Lexems verstanden werden, bestimmte Realisierungsstrukturen zu bevorzugen und auch die lexikalische Besetzung dieser Strukturen maßgeblich zu beeinflussen. Dabei soll die Bestimmung des Valenzträgers als, verbal oder nominal zu realisierend" auf eine Abstraktionsstufe (etwa im Sinne der „basic valency“

${ }^{26}$ Mit dem so gewonnenen strukturellen Imperativkriterium kann man dagegen argumentieren, daß die Formen Essen Sie! und Essen wir! Imperative seien (Engelen (1984), 94). Hier drückt man Sprechakte wie ,Aufforderung', ,Wunsch', ,Bitte' usw. mit Hilfe des Indikativparadigmas aus. Die ungarischen Entsprechungen sind jedoch auch hier Imperative, denn das Imperativparadigma des Ungarischen ist vollständig. Die vierte mathematisch mögliche Gegenüberstellung im Imperativ (Egyél! vs. I $\beta$ !) stellt im Sinne des über die Ellipse Gesagten keine Strukturkorrespondenz dar. 
von Mosel (1984), 3 f.) hindeuten, bei der man zwar weiß, daß eine Verbform, Adjektivform usw. realisiert werden soll, aber es geht noch nicht um tatsächliche Verbformen, Adjektivformen usw. Auf dieser Abstraktionsebene braucht man nominale und verbale Strukturen nich $\mathrm{t} \mathrm{mehr}$ miteinander zu verrechnen, und Prioritäten zwischen den verschiedenen Verbstrukturen, Adjektivstrukturen usw. sind noch nicht festgelegt. Der kategoriale Anteil der verbalen Valenzpotenz, der vom Valenzträger her den strukturellen Realisierungsprozeß steuert, besteht auf der vorgesehenen Abstraktionsebene m. E. vor allen Dingen aus einer Kombination von Kasuskategorien (vgl. 1.4.2.2.).

Bei dem folgenden Versuch, dem oben skizzierten Problem nachzugehen, beschränke ich mich auf die eingeführte Gegenüberstellung ,Deutsch - Ungarisch' und die Realisierung des Erstaktanten.

1.4.1. Die Erscheinungsform des Erstaktanten kann zwar in beiden Sprachen ,Nominativergänzung' (im folgenden: E1) genannt werden, es handelt sich aber um zwei unabhängige Kategorien, deren Tertium comparationis die allgemein-dependenzielle Kategorie ,Erstaktant' darstellt.

Was ist aber genau unter ,E 1' im Deutschen und Ungarischen zu verstehen? Geht es um die Ergänzungen im Nominativ?

Die Frage ist eine doppelte. Denn einerseits wird mit ihr danach gefragt, ob nur derjenige Teil - segmentierbar oder nicht - einer Zwei-EbenenRealisierung, der die nominale Realisierung der funktionalen Nominativkategorie darstellt, als Ergänzung angesehen werden soll. Andererseits geht es um den Status kommutierender Ergänzungssätze und Infinitivkonstruktionen. Da jedoch letzterer unsere Fragestellung unmittelbar nicht betrifft, soll nur darauf verwiesen werden, da $ß$ ich sie nicht zu den Erscheinungsformen der $\mathrm{E} 1$ rechne (vgl. Ágel (1988), 132 ff.).

Schwieriger erscheint mir die andere Frage. Denn einerseits werden am deutchen und ungarischen Nomen verschiedene nominale Kategorien realisiert, andererseits kann jedoch die Redeweise ,das Verb X eröffnet eine Leerstelle nicht so verstanden werden, daß Person und Numerus einer Ergänzung vom Verb abhängen. Da es allerdings aus Raumgründen unmöglich ist, dieses Thema hier weiter zu verfolgen, sollen E 1en ausschließlich auf der Grundlage ihrer Realisierungseigenschaften bestimmt werden. Der Begriff, Valenzstelle für eine E 1' wird so zu interpretieren sein, daß im Verb die Paradigmenkategorie, Nominativ' kodiert ist, was in finiten Äußerungen eine deiktisch-anaphorische Konstituentenrealisierung der nominalen Nominativkategorie bewirkt.

Über die nominale Nominativkategorie hinaus werden an den E 1en beider Sprachen Personen- und Numeruskategorien realisiert. Die nominale Realisierungspotenz der funktionalen Kasuskategorie kann, soweit Finitheit ausgedrückt werden soll, auf Mikro- oder Makroebene zum Tragen kommen. Der „Träger" auf Mikroebene ist der obligatorisch zu realisierende Mikroaktant, also derjenige (oft nicht segmentierbare) Teil des Verbflexivs, der zumindest die verbalen Person- und Numerusinformationen trägt. Der "Träger“ auf 
Makroebene ist der Makroaktant, also die traditionell syntaktisches Subjekt genannte Einheit. Er trägt, soweit realisiert, die nominalen Nominativ-, Personund Numerusinformationen. (Zum sog. Prädikatsnomen vgl. 4.3.).

1.4.2. Auf dem Hintergrund der festgestellten Gemeinsamkeiten können wir uns der Erörterung der strukturellen Unterschiede zwischen den Erstaktantenrealisierungen zuwenden.

1.4.2.1. Als erstes soll geklärt werden, wie der Begriff, strukturelles Deiktikon/ Anaphorikon' (vgl. 1.3.5.3.) im Rahmen einer Theorie des Sprechens zu verstehen ist.

Die Antwort, die hier vertreten wird, ist, daß ein strukturelles Deiktikon/ Anaphorikon keinem besonderen Diskursdruck ( $=$ Druck, dessen Quelle die Textproduktion und/oder -rezeption ist) ausgesetzt werden muß, um seine deiktisch-anaphorische Potenz zu entfalten. Daraus folgt umgekehrt, daß es eines Diskursdrucks bedarf, um die Realisierung dieser Potenz zu blockieren. Es ist genau in diesem Sinne zu verstehen, daß die Verbflexive im Ungarischen strukturell deiktisch/anaphorisch sind, weshalb sie durchgehend als VFLEX $_{+ \text {anaph }}$ symbolisiert werden sollen.

Die Situation im Deutschen scheint nun eine Art Spiegelbild der im Ungarischen zu sein. Die (indikativischen) Flexive können zwar, wie Reduktions- und Aufhebungsformen nahelegen, deiktisiert/anaphorisiert werden, dazu müssen sie aber einem Diskursdruck ausgesetzt werden. Umgekehrt bedarf es keines besonderen Diskursdruckes, um die Realisierung der strukturellen Deiktizität/Anaphorizität der makrovalenziellen Ebene zu überlassen. Deshalb sind die (indikativischen) Verbflexive des Deutschen strukturell nicht deiktisch/anaphorisch: VFLEX -anaph $_{\text {. }}$

Um zu einem Verständnis der unterschiedlichen strukturellen Verhältnisse in Deutsch und Ungarisch zu kommen, reicht es allerdings nicht, auf die unterschiedliche Natur der VFLEX hinzuweisen. Man muß auch die Frage stellen, wie die funktionale Nominativkategorie in beiden Sprachen realisiert wird, welche Konstituentenstrukturen dabei in Erscheinung treten und in welchem Zusammenhang die Nominativrealisierungen mit den beiden VFLEXTypen stehen.

1.4.2.2. Wie kommt eine Verbform zu ihrer Konstituentenkategorie, verbaler Nominativ': Ist die Nominativinformation im Verblexem, im VFLEX oder in beiden kodiert? Und ist es überhaupt adäquat, einer Verbform - egal welcher Sprache - automatisch nur eine verbale Konstituentenkategorie zuzuschreiben?

Für einen GB-Syntaktiker ist die Antwort eindeutig: Der Nominativ wird strukturell zugewiesen, der Kasuszuweiser ist das Finitheitsmerkmal INFL (genauer das AGR(eement)-Element in INFL), d. i. die kategoriale Repräsentation der Kongruenzmerkmale. Und da eine doppelte - lexikalische und strukturelle - Kasuszuweisung redundant wäre, ist im Subkategorisierungsrahmen des Verblexems keine Nominativinformation enthalten. 
Der strukturell-typologisch interessierte Valenzforscher kann auf die obige Redundanzregel m. E. nicht zurückgreifen. Ihn interessiert die Realisierung der einzelsprachlichen Konstituentenkategorien unter dem Aspekt der funktionalen Nominativkategorien. Vergleichen wir dt. er kommt bzw. ${ }^{*}$ er regnet und ital. viene bzw. *lui piove:

Das Problem mit *er regnet - im Vergleich zu er kommt - scheint entweder ein außersprachliches (eines mit dem „Weltwissen“) und/oder ein inhaltliches (keine Agenskodierung) zu sein. Wir können es aber durchaus auch grammatisch interpretieren. Da Person und Numerus korrekt realisiert sind und da es wohl abwegig ist, dem Verb regn- eine Genuskategorie (eine Paradigmenkategorie ,Neutrum') zuzuschreiben, müssen wir annehmen, daß sich die Nominativrektion zwischen er und regnet nicht einstellen konnte. Da durch er die nominale Nominativkategorie vertreten ist, ist die Verletzung der Rektionsbeziehung mit dem Umstand zu erklären, daß regnet - im Gegensatz zu kommt - über keine verbale Nominativkategorie verfügt.

Das Problem mit ${ }^{*}$ lui piove kann jedoch nicht analog sein, denn das Beispiel viene zeigt, daß die Mikroebene -e eine korrekte E 1 darstellt, also die nominale Nominativkategorie korrekt vertritt.

Wenn nun regnet über keine verbale Nominativkategorie verfügt, wobei -et ein VFLEX - anaph $_{\text {ist, }}$ und wenn piove die nominale Nominativkategorie

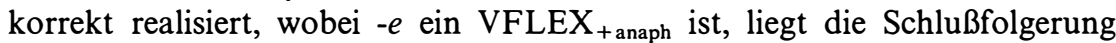
nahe, da ${ }^{*}$ er regnet deshalb inkorrekt ist, weil die realisierte Verbform regnet - im Gegensatz zu kommt - von dem Verblexem regn- - im Gegensatz zu komm- - keine verbale Nominativkategorie zugewiesen bekommt. Umgekehrt ist *lui piove deshalb inkorrekt, weil das realisierte Ver blexem piov- im Gegensatz zu vien- - von dem Verblexem piov- - im Gegensatz zu ven - - keine verbale Nominativkategorie zugewiesen bekommt. Die „äußere“ Nominativrektion (zwischen Pronomen und Verbform) könnte hier jedoch nur dann zustande kommen, wenn die „innere“ (zwischen Verblexem und Mikroebene) korrekt realisiert wäre. Dies ist aber im Falle von piov-, das über keine Paradigmenkategorie ,Nominativ'verfügt, nicht möglich.

Der entscheidende strukturelle Unterschied zwischen einer Sprache wie Italienisch und einer wie Deutsch besteht darin, daß sich die Realisierung der funktionalen Nominativkategorie im Deutschen zwischen Verbform und Makroebene, im Italienischen jedoch verbformintern - zwischen realisiertem Verblexem und Mikroebene - abspielt. In Sprachen mit VFLEX $+_{\text {anaph }}$ besteht die strukturell primäre nominativische Rektionsbeziehung zwischen realisiertem Verblexem und realisierter Mikroebene.

Diese Überlegungen sind mit der traditionellen valenzgrammatischen Annahme konform, daß die E1-Stelle zur Valenz der meisten deutschen Verben gehört (aber eben nur auf der in 1.4. postulierten Abstraktionsebene). Aber auch unabhängig von valenzgrammatischen - oder irgendwelchen ,ideologischen" - Überlegungen sehe ich keinen Widerspruch darin, daß etwa das italienische Finitum seinen Nominativ doppelt zugewiesen bekommt. Ganz im 
Gegenteil: Wenn nämlich der Sprachteilhaber eine Redundanzregel wie die im VFLEX kodierte Nominativinformation einmal beherrscht - wobei diese Formulierung nicht als Stellungnahme hinsichtlich des ontogenetischen Status der Regel zu verstehen ist -, dann beherrscht er sie mit Sicherheit auch auf der Ebene des Valenzrahmens der einzelnen Verben, vorausgesetzt, die Paradigmenkategorien des Verbs widersetzen sich nicht. Denn im konkreten Sprechen macht der Sprecher-Hörer nicht mit separat kodierten Nominativinformationen Erfahrungen, sondern mit an Wortformen realisierten Informationen. Und es ist höchst unwahrscheinlich, daß diese ,idiosynkratischen“ Erfahrungen eine redundanzfreie Informationsspeicherung in Form von sauber getrennten strukturellen und lexikalischen Regeln fördern würden. Fragt man einen deutschen Sprecher, welche Kasusformen er sich in der Umgebung des Verblexems vergeud- vorstellen kann, muß er wohl keinen syntaktischen Algorithmus ablaufen lassen, um diese Frage zu beantworten (vgl. auch Welke (1992)). ${ }^{27}$

$\mathrm{Da} ß$ die verbale Nominativkategorie tatsächlich im Verblexem kodiert ist, haben für das Deutsche die Assoziationsexperimente von Heringer (1985) eindrucksvoll gezeigt. Obwohl sich hier die Versuchspersonen Fragen zu Infinitiven einfallen lassen mußten, kamen die Subjektfragen auf Platz 2.

Darüber hinaus gilt es festzuhalten, daß es funktional in keiner Sprache eine doppelte Kasuszuweisung gibt. Denn die Funktionalität einer Kategorie besteht per definitionem darin, daß alle Realisierungseigenschaften der Kategorie als Funktive derselben Funktion (etwa im Sinne von Hjelmslev) betrachtet werden.

${ }^{27}$ M. E. ist es einer der Irrtümer generativistischer Modellbildung, der sich auf das Naturwissenschaftlichkeitspostulat der Theorie zurückführen läßt, redundanzfreie Modelle zu bevorzugen und zu glauben, solche Modelle könnten Kandidaten für die Erfassung sowohl des angeborenen wie auch irgendeines aktuellen Sprachvermögens sein. Daraus bzw. der autonomen Syntaxauffassung folgt die nichtexplizierte, jedoch offensichtliche Annahme, daß Redundanz eine absolute Größe sei: Was von dem Gesichtspunkt $\mathrm{X}$ aus redundant ist, müsse auch von allen anderen nur denkbaren Gesichtspunkten aus redundant sein (zu diesem Problem vgl. Ágel (1991)). Und obwohl die „Betrachtung der Grammatik aus der Perspektive des Lexikons [...] eine spezifische und originäre Position der Valenzgrammatik“ ist (Welke (1992)), obwohl daher valenzielle Lexikoneinträge durchaus redundanter(!) sein sollten und „dürften“ als generativistische, ist dies trotz struktureller Kasuszuweisung nicht unbedingt der Fall. Ich denke nicht nur daran, daß in der GB auch mit passivischen Lexikoneinträgen gearbeitet wird und daß Thetarollen (semantische Kasus), die eine die elementare Satzstruktur modellierende Bedeutungsangabe (Ágel (1988), $161 \mathrm{f}$. und $169 \mathrm{ff}$.) überflüssig machen kann, angegeben werden müssen, sondern auch daran, $\mathrm{da} ß$ in einem GB-Lexikoneintrag auch die strukturelle Kasusrealisierung repräsentiert sein muß, um sicherzustellen, daß der Kasus seine NP findet (vgl. Grewendorf (1991), $151 \mathrm{f}$.). (Dabei sieht man sich mit der intuitiv(!) gewiß nicht einfachen Aufgabe konfrontiert, sich kasuslose NPn vorstellen zu müssen.) Wird auch der Dativ als struktureller Kasus angesetzt (Wegener (1990), $160 \mathrm{ff}$.), werden die Lexikonverhältnisse womöglich auch nicht einfacher, denn dann müssen die möglichen ThetarollenZuordnungen der strukturellen Kasus im Lexikon auseinandergehalten werden. 
1.4.2.3. Wenden wir uns nun dem Unterschied zwischen Ungarisch und Deutsch zu:

(1) Im Sinne des über das Italienische Gesagten muß im ungarischen VFLEX die nominale Nominativinformation kodiert sein. Da das ungarische Verb kraft der Opposition ,definite vs. indefinite Konjugation“ über die Einheitenkategorie ,Nominativ' verfügt (1.3.1.), haben wir für diese Entscheidung im Falle des Ungarischen ein zusätzliches Argument. Dabei ist - wie oben gezeigt - das Wesentliche an einer im Verbflexiv kodierten Nominativinformation, daß es strukturell ausreicht, sie am Verb zu realisieren. Die Realisierung des Verbflexivs ist sozusagen auf die des Verblexems - und nicht auf die einer externen Konstituente - gerichtet. Die kodierte Information übt keinen Druck auf die makrovalenzielle Realisierung aus.

(2) Da demgegenüber in den (indikativischen) VFLEX des Deutschen keine Nominativinformation kodiert ist, sind - im Gegensatz zum Ungarischen - die strukturellen Realisierungsrichtungen von Verblexem und -flexiv nicht aufeinander gerichtet, sondern das Flexiv setzt die Realisierungsrichtung der lexikalischen Nominativinformation fort. Folglich kommt die Nominativinformation in der realisierten Verbform nicht „zur Ruhe“, sondern sie ist nach außen, auf die makrovalenzielle Ebene gerichtet. Wird dieser Druck auf die makrovalenzielle Ebene nicht durch Diskursdruck neutralisiert, muß ,Nominativ' auch makrovalenziell realisiert werden. Ein wesentlicher Definitionszug der Obligatheit der Mikroebene besteht bei Sprachen ohne in den VFLEX kodierte Nominativinformation also gerade darin, daß die Information weitergegeben werden muß, wenn sich ihr kein besonderer Diskursdruck ,entgegenstemmt".

Schematisch kann der Unterschied zwischen der ungarischen und der deutschen Nominativrealisierung wie folgt zusammengefaßt werden (ung. élek, [ich] leb-e'):

\begin{tabular}{|c|c|c|c|c|c|}
\hline Sp. & i & Verblexem & 1 & Mikroebene & Makroebene \\
\hline Dt. & 1 & leb ,Nominativ ${ }^{6} \rightarrow$ & 1 & $\ldots$ & I +- ich ,Nominativ \\
\hline Ung. & 1 & él ,Nominativ'.$- \rightarrow$ & | & - ek ,Nominativ“ & 1 \\
\hline
\end{tabular}

Diese Darstellung zeigt zugleich analytisch, worin der Unterschied zwischen einer Rektionsbeziehung (zwischen Verbform und Makroebene im Deutschen) und einer Kongruenzbeziehung (zwischen Verbform und Makroebene - soweit letztere realisiert - im Ungarischen), der ja im Italienischen die erwähnte „äußere“ Rektionsbeziehung entspricht, besteht. Ebenfalls analytisch zeigt sie den Unterschied zwischen verbforminterner (Ungarisch) und -externer Rektionsbeziehung (Deutsch).

Die Darstellung und das Gesagte legen jedoch nahe, daß es hier gar nicht um interne und externe Rektionsbeziehungen, sondern um unterschiedliche Konstituentenstrukturen geht: 


\begin{tabular}{|c|c|c|c|c|c|c|c|}
\hline $\mathrm{Sp}$ & । & Verblexem & । & Mikroebene & I & \multicolumn{2}{|c|}{ Makroebene } \\
\hline Dt. & I [ & & lebe & & ] 1 [ & ich & ] \\
\hline Ung & I [ & él & ] I [ & ek & ] 1 & & \\
\hline
\end{tabular}

Die Eisenbergschen Relationen sind damit typologisch adäquat wiederhergestellt.

1.4.2.4. Somit sind die Voraussetzungen für die Beschreibung der Ebenenverhältnisse als ein dynamisches Wechselspiel zwischen Lexikon und Grammatik - inklusive einer dynamischen Konstituentenstruktur - geschaffen:

(1) Im Ungarischen ist die Grammatik (Verbmorphologie) verantwortlich für die Bereitstellung des mikrovalenziellen „anaphorischen Bettes“ für E1. Die VFLEX $_{+ \text {anaph }}$ enthalten die Information, (nominaler) Nominativ'. Ist beim Verblexem die Lexikoninformation, Nominativergänzung' kodiert, ist es durch die Interaktion (Aufeinandergerichtetsein) von realisiertem Verblexem und VFLEX sichergestellt, daß die korrekte Realisierung der funktionalen Nominativkategorie zustande kommt.

Im Falle einer pronominalen makrovalenziellen Realisation geht es um die - durch einen besonderen Diskurssog bedingte - Auslagerung des ,anaphorischen Bettes" auf die makrovalenzielle Ebene. Die Deanaphorisierung des VFLEX geht mit der Abgabe der nominalen Nominativkategorie an das Personalpronomen einher. Wird die makrovalenzielle Ebene lexikalisch besetzt, geht die Anaphorizität von VFLEX in der Referenz des Lexemwortes auf.

(2) Im Deutschen ist die Situation etwas komplizierter:

Bei indikativischer (und konjunktivischer) Verbrealisierung ist die Grammatik (Verbmorphologie) verantwortlich dafür, daß wegen der fehlenden Anaphorizität von VFLEX ein pronominales, makrovalenzielles „anaphorisches Bett" bereitgestellt wird. Ist im Valenzrahmen des zu realisierenden Verblexems eine E1 kodiert, erfolgt die Weitergabe dieser Information an die Verbform. $\mathrm{Da}$ die Information gerichtet bleibt und da wegen VFLEX $_{\text {-anaph }}$ die Weiterleitung nicht abgeblockt wird, kann eine korrekte strukturelle Realisierung der funktionalen Nominativkategorie nur dann zustande kommen, wenn auch die Makroebene realisiert wird.

Ist im Valenzrahmen keine E 1 vorgesehen, kann keine Information, E 1 oder ,Nominativ' an die Verbform weitergeleitet werden. Ein Verblexem wie regn- „fusioniert“ also mit seinem diskontinuierlichen Allomorph es ...-et (vgl. auch 2.), die Einheit es regnet kann jedoch keine realisierte E1 beinhalten, denn in regnet ist keine verbale Nominativinformation enthalten. Selbst wenn man es nominativisch interpretiert (vgl. jedoch 2.2. und Anm. 32), ist also in es regnet die funktionale Realisierung der Nominativkategorie nicht gewährleistet.

Im Falle einer lexikalischen Besetzung der makrovalenziellen Ebene wird - im Gegensatz zu (1) - keine zusätzliche Interaktionskomponente zwischen 
den beiden Ebenen anzunehmen sein, da sich die oben dargestellte „Entstehung der Referenz" innerhalb der makrovalenziellen Ebene abspielt.

Bei imperativischer Verbrealisierung wird demgegenüber kraft der Anaphorizität von VFLEX ein mikrovalenzielles „,anaphorisches Bett“ bereitge-

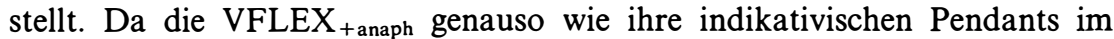
Ungarischen und Italienischen über die Information ,Nominativ' verfügen müssen, besteht hier eine Rektionsbeziehung zwischen realisiertem Verblexem und Mikroebene. Somit ist die (durch die Nominativfunktion begründete) Konstituentenstruktur im deutschen Imperativ mit der im ungarischen oder italienischen Indikativ identisch. Die Verbform allein stellt eine korrekte Realisierung der funktionalen Nominativkategorie dar.

Der Imperativ interagiert mit einer verbalen Personenkategorie ,2. Person", die von den sonst nominalen Personenkategorien des Deutschen (vgl. 1.3.5.2.) strikt zu unterscheiden ist. Die Annahme einer für alle Sprachen geltenden Kategorie ,nominale Person' (oder eben ,verbale Person') wird demnach nicht nur durch den strukturellen Vergleich von Sprachen, sondern auch angesichts einzelsprachinterner Strukturmischungen in Frage gestellt.

Während die Besetzung der makrovalenziellen Ebene im Ungarischen auf einen Diskurssog zurückgeführt wurde, ist ihre Nichtbesetzung im Deutschen mit einem Diskursdruck zu erklären: Dort bewirkt der Diskurs (inklusive syntaktisch-lexikalischer E 1-Realisierungen), daß die Struktur ,gedehnt“ wird, hier bewirkt er, daß sie komprimiert wird. Der grammatische Niederschlag dieser entgegengesetzten Bewegungen ist ein „Austausch“ der Konstituentenstrukturen (ung. én, ich'):

\begin{tabular}{|c|c|c|c|c|c|}
\hline & 1 & Mikroebene & I & Makroebene & I Diskurs \\
\hline [ & leb ] I [ & e & ]$+\ldots$ & $\ldots$ & .... Druck \\
\hline[ & élek & & ] 1 [ & én & ] $\ldots \rightarrow$ Sog \\
\hline
\end{tabular}

Die der strukturell normalen Rektionsbeziehung des Deutschen entsprechende Kongruenzbeziehung (bzw. die entsprechenden Rektionsbeziehungen im Italienischen, Spanischen usw.) entsteht im Ungarischen nur unter Diskurssog.

Demgegenüber ist die strukturell normale Rektionsbeziehung des Ungarischen im Deutschen nur unter Diskursdruck möglich, d. h., „ungarische“ verbforminterne Verhältnisse können im Deutschen nur grammatisch-kom-

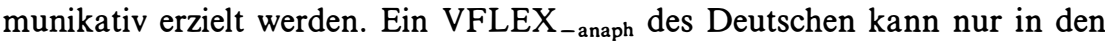
verschiedenen grammatisch-kommunikativen Reduktions- und Aufhebungsformen (z. B. riecht gut, vgl. 2. und 5.) als alleiniger (mikrovalenzieller) Träger der E1-Qualität in Erscheinung treten. Daher muß man annehmen, daß der grammatische Gehalt des „erfolgreichen“ Diskursdruckes darin besteht, das VFLEX $_{\text {-anaph }}$ nominativisieren und a naphorisieren zu können. Für die 
Interpretation von ,Nominativisierung und ,Anaphorisierung' kommen dabei zwei Möglichkeiten in Frage:

(1) Der Diskursdruck ersetzt (vertritt) die nominalen Konstituentenkategorien ,1. Person', ,Singular' und ,Nominativ'. Es handelt sich also nur um eine scheinbare Nominativisierung und Anaphorisierung des VFLEX. Diese Lösung widerspricht jedoch dem oben postulierten „Austausch“ der Konstituentenstrukturen.

(2) Der Diskursdruck bewirkt, daß die Realisierung der nominalen Konstituentenkategorien ,1. Person', ,Singular' und ,Nominativ' in die Mikroebene komprimiert wird. Dadurch wird das VFLEX tatsächlich nominativisiert und anaphorisiert. Ich plädiere für diese „grammatischere“ Lösung, ohne jedoch (1) für abwegig zu halten.

Damit haben die Begriffe ,Mikroebene' und ,Makroebene' eine neue, dynamische Interpretation erfahren. Sie sind komplementäre Erscheinungsformen der E1 (vgl. auch Ágel (1991)). Pasierbskys Begriff der Repräsentation (vgl. Anm. 12 und 1.3.5.3.) wäre also dahingehend zu modifizieren, da $\beta$ in Sprachen wie Deutsch bei Modifikationen die Makroebene durch die Mikroebene repräsentiert wird. Umgekehrt wird in Sprachen wie Italienisch oder Ungarisch bei Modifikationen (inklusive syntaktisch-lexikalischer E 1-Realisierungen) die Mikroebene durch die Makroebene repräsentiert. Die Kategorie ,Zwei-Ebenen-Aktant' ist daher auf die Systemebene zu beziehen. Dabei bleibt der Primat der Mikroebene im Sinne einer Vorkommensbedingung unangetastet.

1.4.3. Die obige Darstellung kann natürlich nicht auf alle Sprachen der Erde angewandt werden, möglicherweise nicht einmal auf alle Nominativsprachen. Mutatis mutandis, d. h. unter Einbeziehung zusätzlicher, einzelsprachlich relevanter Beschreibungskategorien, kann sie jedoch für eine Reihe von Sprachen (z. B. Latein, Spanisch, Italienisch, Ungarisch, Finnisch, Serbokroatisch, Polnisch, Portugiesisch usw. „versus“ Deutsch, Englisch, Französisch, Russisch, Schwedisch usw.) eine typologisch orientierte, strukturelle Beschreibungsgrundlage bieten. Dabei deuten nicht nur die obigen Überlegungen, sondern auch einschlägige Analysen von Einzelsprachen in GB und DG darauf hin, $\mathrm{da} ß$ man in den weiteren Untersuchungen insbesondere drei Umstände zu berücksichtigen hat:

(1) Mit einer komplexen funktionalen Kategorie ,Person' (und wahrscheinlich auch ,Numerus') kann man der „Buntheit“ einzelsprachlicher Strukturen nicht gerecht werden. Dies ist auch in dem Sinne zu verstehen, daß man ohne einzelsprachliche Realisierungsevidenzen mit keinem paradigmatischen Zwang rechnen kann, der einfache funktionale Kategorien wie ,1. Person', ,2. Person' und 3. Person' (evtl. zusätzlich nach Modus getrennt) beinhaltet (vgl. etwa das Finnische (Järventausta (1991), $177 \mathrm{ff}$.) und umgekehrt das Deutsche in 1. und 2. Person Singular Indikativ).

(2) Möglicherweise ist es ein Ei-Huhn-Problem, ob in Sprachen mit E 1 reiche(!) Konjugationssysteme die notwendige Voraussetzung für verbformin- 
tern realisierbare nominativische Rektionsbeziehungen oder ob diese die notwendige Voraussetzung für Strukturen mit durchgehenden VFLEX ${ }_{+ \text {anaph }}$ darstellen. Vergleicht man verschiedene Flexionssysteme (etwa das deutsche mit dem irischen (Jaeggli/Safir (1989), 29 f.), kann man sich leicht davon überzeugen, daß der Begriff des Reichtums höchstens nur funktionalsprachlich interpretiert werden kann. In diesem Zusammenhang soll auch auf László (1988), 220 hingewiesen werden. Sie erklärt den Umstand, daß die Defektivität eines ungarischen Konjugationsparadigmas keine makrovalenziellen „Gegenmaßnahmen“ bewirkt, so, „daß das Paradigma für den Linguisten, nicht aber für den Sprecher defektiv ist." Vgl. auch die GB-theoretischen Untersuchungen der „no agreement“-pro-drop-Sprache Chinesisch (bzw. Koreanisch und Japanisch) in Huang (1984) bzw. (1989).

(3) Es ist zweckmäßig, strukturelle Untersuchungen von Valenzrealisierungen im Rahmen einer allgemeinen ,head-marking“-,,dependent-marking“Typologie Nicholsscher Art durchzuführen, denn erst ein komplexes Bild über die generellen strukturellen Realisierungsverhältnisse der untersuchten Einzelsprache (im Kontrast zu denen anderer Einzelsprachen) macht eine adäquate Einordnung der Valenzrealisierungsstrukturen und eine adäquate Einschätzung dessen, was die relevanten Beschreibungskategorien sein können, die für die Interaktion mit diesen in Frage kommen, möglich. Einen Ansatz in diese Richtung stellen die Überlegungen in László (1988), 230 f. dar.

1.4.4. Aufgrund der Darstellung in 1.4.2. sollte es verständlich geworden sein, warum hier trotz gewisser Ähnlichkeiten (z. B. nominaler Charakter der Mikroebene in Ungarisch oder Italienisch - allerdings nur, wenn die Makroebene nicht realisiert ist) nicht versucht wurde, die verschiedenen pro-Theorien der GB (vgl. z. B. Rizzi (1986) und die Aufsätze in Jaeggli/Safir (Hg.) (1989)) in die valenzgrammatische Beschreibung zu integrieren. Zwar differieren diese Theorien erheblich voneinander, sie richten sich jedoch nach gewissen Grundannahmen der GB-Theorie (empty category principle [ECP], Erweitertem Projektionsprinzip [EPP], Theta-Kriterium), die mit der hier vertretenen Auffassung nicht konform sind.

Im Sinne des EPP, dessen universaler Status allerdings umstritten ist (Haider (1991), 52), geht man davon aus, daß ,all clauses must contain subjects at all syntactic levels of representation. Crucially, it is claimed that this state of affairs holds regardless of the particular nature of the subject [Hervorhebung - V. Á.], so that subject position is argued to be present whether it has phonological or semantic content or not. Thus, a subject may be phonologically null or overt, and it may be a meaningfull argument of the verb or be semantically vacuous." (Franks (1990), 2) Da jedoch in bestimmten Konstruktionen auch keine pro-drop-Sprachen (wie Deutsch) das phonologische Null-Subjekt zulassen (weil pro getrunken wird bzw. wenn man auch das nichtrealisierte Passivsubjekt berücksichtigt: weil pro pro getrunken wird), unterscheidet Rizzi (1986) zwischen formaler Lizensierung und inhaltlicher 
Determination des pro. Der signifikante Unterschied zwischen Ungarisch und Deutsch wäre demnach vereinfacht wie folgt beschreibbar:

Im Ungarischen kann der Flexionskomplex INFL das leere Subjektpronomen pro formal lizensieren, d. h. ihm Nominativkasus, Person bzw. Numerus zuweisen, wobei pro auch inhaltlich determiniert (referentiell) ist, so $\mathrm{da} ß$ sich die Setzung eines lexikalischen Pronomens (= pronominalen Makroaktanten) erübrigt. Im Deutschen ist die formale Lizensierung zwar möglich, nicht aber die inhaltliche Determination. Folglich ist im Interesse der Sicherung der Referentialität der Subjektposition die Setzung eines lexikalischen Pronomens im Normalfall notwendig. Im Falle von weil getrunken wird wird das Fehlen des lexikalischen Pronomens - das erste pro oben - dadurch erklärt, daß pro inhaltlich gar nicht determiniert (kein Argument) sein könnte, denn ein passivischer Lexikoneintrag (getrunken) kann der Subjektposition keine Thetarolle zuweisen (Semi-pro-drop-Sprache).

Das es impersonale der Wetterverben paßt weiterhin nicht in dieses Bild - es müßte ja weglaßbar sein -, wird also von Safir $(1985,215 \mathrm{f}$.) in Anlehnung an Chomsky als Quasi-Argument (idiomatisch spezifisches Argument mit Thetarolle) eingeordnet. (Demgegenüber bleiben Fanselow/Felix (1990 a, 79) bei dessen „non-argument“-Status.) Des weiteren wird die ursprüngliche Eleganz des pro-drop-Parameters - und des ECP - dadurch getrübt, daß leere Subjekte im Deutschen dem ECP widersprechen, weshalb Safir $(1985,209)$ die Ausnahme macht: „The ECP does not apply to pronominals". Auch liegt es nahe, in Fällen wie Mich friert oder Mir graut mit einem pro rechnen zu müssen (Grewendorf (1991), $146 \mathrm{f}$.), was zusätzliche Komplikationen impliziert (vgl. etwa Shin (1988), 82 f.). Im Endeffekt unterscheiden sich also die GB-Erklärungen von dem traditionellen Konzept des formalen Subjekts nur dadurch, daß es auch phonetisch leere formale Subjekte gibt, die dann aufwendig motiviert werden müssen.

Was den Leser von Pasierbsky, Nichols und László an dieser Lösung zusätzlich, sozusagen generell stört, ist deren offensichtliche „dependent-marking orientation" (vgl. auch Anm. 22): INFL in Sprachen mit durchgehend VFLEX $_{+ \text {anaph }}$ wird nicht als (auch) Subjektträger anerkannt, sondern seine diesbezügliche Potenz wird auch in diesen Sprachen in die Subjektposition ausgelagert (selbst wenn u. U. für eine VP-intern generierte Subjektposition im Ungarischen argumentiert wird, um u. a. gerade dem ECP Rechnung zu tragen, vgl. É. Kiss (1987), 26 ff.). Auf diese Weise wird eine sich aus theorieinternen Prinzipien ergebende, sich durch eine relativ statische Konstituentenstruktur manifestierende Parallelität zwischen Sprachen wie Italienisch (Ungarisch) und Deutsch etabliert. Dabei bedarf insbesondere die Beschreibung von Modifikationen und einzelsprachinternen, nichtdialektalen Strukturmischungen (deutscher Imperativ) einer dynamischen Konstituentenstruktur. Einen nichtkanonischen Weg geht jedoch Haider (1991).

In dem in 1.4.2. vorgeschlagenen theoretischen Rahmen bedarf eine Struktur wie weil getrunken wird (oder eben Hier wird getrunken, das in der GB 
wiederum anders als weil getrunken wird interpretiert werden muß) keiner besonderen Erklärung. Am „normalen“ (nicht adressatenpassivischen) passivischen Finitum kann im Deutschen nur dann eine verbale Nominativkategorie realisiert sein, wenn das entsprechende aktivische über eine verbale Akkusativkategorie verfügt (vgl. Hier wird Bier getrunken). Wenn dem nicht so ist (Hier wird getanzt), besteht vom realisierten Verblexem bzw. von der (nichtnominativischen) passivischen Verbform her auch kein Druck auf die nominale Realisierung der Nominativkategorie, es wird also weder formal noch inhaltlich eine E 1 realisiert. (Das Problem der Opposition, Hier wird getanzt vs. Es wird getanzt' ist ein topologisches (vgl. Anm. 34 und 37). Die Konstruktion *Hier wird es getanzt ist für Haider $(1991,59)$ ein Argument gegen eine obligatorische strukturelle Subjektposition im Deutschen. $)^{28}$

Es ist eine mögliche Konsequenz des in diesem Abschnitt Gesagten, daß ein für viele Wissenschaftler beschreibungsadäquater Vorschlag wie der prodrop-Parameter der GB von einem anderen Gesichtspunkt aus „nicht so intuitiv" wirkt (vgl. auch 4.2.). Da Chomsky die generative Theorie ohnehin mit der Chemie verglichen hat (vgl. Anm. 2), erscheint mir daher die folgende Parabel aus Kuhn (1976), 64 als ein angemessenes Resümee der Vergleichbarkeitsdiskussion (wenn man will: zwischen „Autonomisten“ und „Funktionalisten“): „Ein Forscher, der etwas darüber zu erfahren hoffte, wie die Wissenschaftler die Atomtheorie auffassen, fragte einen ausgezeichneten Physiker und einen hervorragenden Chemiker, ob ein einzelnes Heliumatom ein Molekül sei oder nicht. Beide anworteten ohne zu zögern, doch ihre Antworten waren verschieden. Für den Chemiker war das Heliumatom ein Molekül, da es sich in bezug auf die kinetische Theorie der Gase wie ein solches verhielt. Für den Physiker war das Heliumatom jedoch kein Molekül, weil es kein Molekülspektrum zeigte.“

1.5. Exkurs: Bevor im folgenden (Abschnitte 2-6.) einige Anwendungen des Zwei-Ebenen-Modells diskutiert werden, soll noch die Frage gestellt werden, ob die strukturell-typologisch orientierte Valenzforschung der Zukunft mit dem Zwei-Ebenen-Modell auskommen wird. Eine Antwort wird anhand des Englischen (vgl. Emons (1978), 30 f.) gesucht:

[1'] My friend writes a letter to her.

[1"] My friend writes her a letter.

${ }^{28}$ Ein funktionaler Ansatz, für den sich das deutsche (und auch das lateinische) Passiv als eine vom Täter abgewandte Diathese (Weisgerber) von den Passiven z. B. der romanischen Sprachen oder des Englischen deutlich unterscheidet (Coseriu (1987 a), 184), hat auch keine Schwierigkeiten, die Opposition es wird geschlafen (oder dormitur) vs. ${ }^{*}$ es wird geregnet (oder * pluitur) zu erklären, obwohl ja das Verb schlafintransitiv ist. Funktional geht es im deutschen Passiv um die „Abwendung“ von einem „Täter" (um dessen Dethematisierung), was sich als eine Art Folgeerscheinung formal in der „Nominativisierung“ der aktivischen Akkusativergänzung ausdrücken kann. 
Der Valenzforscher des Englischen ist hier mit dem Problem konfrontiert, ob er to her in [1'] und her in [1"] als Elemente derselben oder von zwei verschiedenen Ergänzungskategorien zu identifizieren hat. „Germanistisch gesehen" scheint alles klar zu sein: Die zwei Elemente sind an der gleichen Position nicht kommutierbar und auch unterschiedlich - eben durch to her bzw. her - anaphorisierbar (etwa im Sinne von Engel (1982), 164 ff.). Demnach müßten sie als Elemente von zwei verschiedenen Ergänzungskategorien angesehen werden.

Die traditionelle germanistische Sicht der Dinge, d. i. die Zugrundelegung des rein syntaktischen Valenzmodells, erweist sich auch hier als unzulässig. Denn erstens ist der inhaltliche Unterschied zwischen [1'] und [1"] am adäquatesten als ein Unterschied in der Funktionalen Satzperspektivierung und nicht als einer in der (Sachverhalts-)Darstellung beschreibbar. Ansonsten müßte man nicht nur im Falle von write, sondern auch bei allen englischen Verben mit der obigen Realisierungsalternative jeweils zwei Verbvarianten annehmen (Emons (1978), 30). Darüber hinaus exkludieren to her und her "jeweils in einem Satz, auch wenn sie nicht an der gleichen Stelle stehen" (Emons, ebd.), sie sind also nicht unabhängig voneinander: Man kann to her und her mit Recht als (auch) topologische Varianten derselben Ergänzungskategorie auffassen. Auch Emons setzt hierfür eine Ergänzungskategorie (E 4) an.

Wertet man nun dieses Ergebnis im Lichte des Zwei-Ebenen-Modells aus, liegt die Fragestellung nahe, ob uns Mikro- und Makroebene ausreichen, um die englischen Ergänzungskategorien strukturell-typologisch adäquat zu charakterisieren, oder anders formuliert: $\mathrm{Ob}$ die Topologiegebundenheit einer Ergänzungskategorie als eine mögliche Eigenschaft eines Makroaktanten anzusehen oder ob für sie eher eine dritte Valenzrealisierungsebene anzusetzen ist. Es geht hier, wohlgemerkt, nicht darum, ob die Funktionale Satzperspektivierung in eine strukturelle Valenzbeschreibung einzubeziehen ist oder nicht. Es geht um den valenztheoretischen Status von Wortstellungskonstellationen, die Aktantenrealisierungen strukturell (mit)begründen.

Eine ausreichend fundierte Antwort ist für den Germanisten nicht möglich. Angesichts der Tatsache jedoch, daß es einerseits reine head-markingSprachen wie Abchasisch oder Navaho gibt, deren Makroaktanten wohl rein topologisch beschrieben werden müßten, und da $ß$ es andererseits reine dependent-marking-Sprachen wie z. B. Japanisch gibt, deren Makroaktanten formal ausreichend charakterisiert werden können, scheint es erwägenswert, trotz der funktionalen Abhängigkeit topologischer und formaler Eigenschaften von Aktanten, die ja im Falle der Emonsschen E 4-Kategorie die starke Form der Komplementarität annimmt, für spätere Untersuchungen eine dritte - topologische - Valenzrealisierungsebene ins Auge zu fassen. Um Mißverständnissen vorzubeugen: Auf dieser strukturellen Realisierungsebene wären nicht die topologischen Eigenschaften einer Sprache $\mathrm{L}_{1}$ zu beschreiben, denn das kann und soll nicht die Aufgabe eines Valenzansatzes sein, sondern nur topologische 
Eigenschaften einer $L_{1}$, ohne Bezug auf die eine oder mehrere Ergänzungskategorien von $\mathrm{L}_{1}$ adäquat nicht definierbar sind. Dabei kann die Art der valenz(realisierungs)relevanten topologischen Eigenschaften von Sprache zu Sprache und auch innerhalb derselben Sprache variieren. Die möglichen Arten wären dann als unterschiedliche Formen von inhaltlichen Beziehungen zwischen den Ebenen dieses Drei-Ebenen-Modells beschreibbar. Bei der graphischen Wiedergabe der Vorkommensrelationen würde es im Anschluß an die Darstellungsmethode in 1.3.7. ausreichen, die dritte Ebene durch die Alternative „0“ oder " $\mathrm{T}_{\mathrm{n}}$ “ [ = topologische Realisierungsebene für die $\mathrm{E}$ (rgänzungskategorie) ${ }_{n}$ vorhanden] zu charakterisieren (z. B. E4 des Englischen: (Mikroebene:) 0; (Makroebene:) $\mathrm{A}_{4}$; (topologische Ebene:) $\mathrm{T}_{4}$ ).

\section{Es impersonale und das Zwei-Ebenen-Modell}

In einer früheren Arbeit $(1988,91 \mathrm{ff}$.) habe ich dafür argumentiert, daß das es der unpersönlichen Verben Bestandteil des Allomorphs des Morphems ,Singular 3. Person“ ist. Bezogen auf ein Verblexem wie regn- heißt das, daß es über die Paradigmenkategorien ,Singular' und ,3. Person' verfügen muß. Ich glaube, diese Lösung ist sowohl mit dem Zwei-Ebenen-Modell der Valenzrealisierungen wie auch mit der vorgeschlagenen Interaktionsbeschreibung der Kategorienrealisierungen gut verträglich.

2.1. Die Frage, der es nachzugehen gilt, ist, ob das es impersonale (also die Pützsche Endmenge 8 oder Typ 10 [formelles Subjekt/Objekt] in Askedal (1990)) Aktantenstatus hat oder nicht. Entscheidet man sich, wie die meisten Valenztheoretiker (beispielsweise Tesnière, Heringer, Engel, Helbig), aus guten Gründen (Nichtreferentialität, Nichtkommutierbarkeit) für "nicht“, ist das Problem immer noch nicht gelöst, denn man hat nur gesagt, was es nicht ist. Man behilft sich mit Etikettierungen wie „uneigentliches Subjekt“, "Scheinsubjekt", „formales Subjekt (Objekt)“, „, formaler Aktant" und dergl., diese drücken im Prinzip aber nur eines aus: So richtig loslösen von der „Dochirgendwie-Subjekt(-Objekt)haftigkeit" des es kann/will man sich nicht. ${ }^{29}$ Aber handelt es sich hier wirklich um eine nichtprototypische Form von Aktanten?

Vergleicht man die Behandlung des Imperativ-Problems mit der des esProblems, können einem valenzgrammatische Zwitterauffassungen recht kurios erscheinen: Auf der einen Seite soll der „klare“ Fall der Valenzreduzierung, also der „,zweifelsfreien“ Subjektlosigkeit sein, wo doch der Imperativ eindeutig subjekthaltig (nominativisch und deiktisch-anaphorisch) ist; auf der anderen Seite der Problemfall, wo doch das es, ebenfalls eindeutig, nichtreferentiell (also auch: nichtdeiktisch-nichtanaphorisch) ist. Wie oben ausgeführt, verfügt

29 Umgekehrt spricht z. B. Engelen $(1986,101)$, der das es impersonale als Subjekt rechnet, von einer „Sonderform des Subjekts“. Vgl. auch das „Quasi-Argument“ der GB. 
jedoch eine E 1 über eine deiktisch-anaphorische Potenz. ${ }^{30}$ Daher kommt es, $\mathrm{da} ß$ es selbst in einem rein syntaktischen Valenzmodell methodologisch unzulässig ist, Minimalparadigmen wie Es regnet: Die Wolken wollen nicht regnen und Es schneit: Dicke Flocken schneiten vom Himmel (Järventausta (1991), 61) heranzuziehen, um für den Subjektstatus des es zu plädieren. Hier fehlt das Tertium comparationis (der Anaphorizität), was nebenbei eine erste Erklärung für das Unbehagen der Muttersprachler über solche Gegenüberstellungen darstellt. ${ }^{31}$

2.2. Die Quelle dieses Unbehagens ist für unsere Betrachtungen insofern wichtig, als (auch) sie Aufschluß über den genauen Status des es impersonale und die Brauchbarkeit des Zwei-Ebenen-Modells geben kann. Kontrastierungen wie Nicht es schneit, sondern dicke Flocken oder Nicht es donnert, sondern ein Motor möchte ich in drei Schritten wie folgt interpretieren:

(1) Ein es in es schneit oder es donnert (zuerst ohne Kontraststellung) kann keine makrovalenzielle Leerstelle besetzen, da hier wegen der E1-losen Lexikoneinträge keine verbalen Nominativinformationen transportiert werden können: schneit und donnert enthalten keine verbale Nominativinformation. Demnach kann auch keine kasuelle Rektionsbeziehung zwischen es und schneit bzw. donnert zustande kommen, folglich kann es auch keinen Nominativ enthalten, der als Bestandteil einer E1 interpretiert werden könnte. (Das Pützsche Beispiel in Anm. 30 ist demnach kein Beispiel für Subjektsanhebung, sondern für eine Akkusativzuweisung durch hör- und die A. c. I.-Konstruktion an ein ansonsten nominativisch-akkusativisches Element. ${ }^{32}$ )

(2) dicke Flocken und ein Motor besetzen in dicke Flocken schneien und ein Motor donnert (ohne Kontraststellung) E 1-Stellen von schnei- und donnermakrovalenziell. Es handelt sich - im Gegensatz zu (1) - um einwertige Verbvarianten von schnei- und donner-.

${ }^{30}$ Obwohl Referentialität als maßgebendes Kriterium natürlich auch von es-Forschern, die nicht aus einem Valenzrealisierungsmodell heraus argumentieren, anerkannt wird, betrachten sie das es impersonale vielfach als Subjekt/Objekt: Pütz (1986, $120 \mathrm{f}$.) aufgrund der Möglichkeit von sog. Subjektsanhebungen (z. B. Peter hört es donnern), Askedal $(1990,218)$ „aufgrund lexikalischer Subkategorisierungsregeln“.

31 Auch Pütz $(1986,120)$ hält die Substitution von es in es klopft durch ein Mann in ein Mann klopft für „willkürlich“. Anderer Meinung ist Askedal (1990, 219). Nach ihm sind bei unpersönlichen Verben wie klopfen, riechen, tropfen „,bei grundsätzlich gleicher Verbbedeutung vollsemantische Subjekte für es substituierbar [...].“

32 Das in 1.4.2.2. Gesagte gilt auch hier: Wir können nicht so tun, als ob syntaktische Funktionen von ihren (im weitesten Sinne) lexikalischen Trägern unabhängig sind. Nur weil das es hier kein Träger der funktionalen Nominativkategorie (und dementsprechend der E1-Kategorie) sein kann, ist es nicht gleich kasuslos, sondern entsprechend seinem neuhochdeutschen Lexikoneintrag nominativisch-akkusativisch. In der A. c. I.-Konstruktion wird seine Akkusativpotenz funktionalisiert. Daher ist die sog. Subjektsanhebung kein „Beweis“ dafür, daß am es bereits vor der Anhebung die nominale Konstituentenkategorie der funktionalen Nominativkategorie realisiert wurde. 
(3) Treten nun es schneit oder es donnert in Kontraststellungen wie in unseren Anfangsbeispielen auf, kann die jeweils zweite Setzung der Verben wegen der grammatischen Konstruktion nicht ... sondern erspart werden (grammatische Reduktion). Dabei besteht der Diskurseffekt darin, daß es okkasionell (,von hinten“) nominativisiert (und zugleich anaphorisiert) wird, d.h. es erfolgt eine okkasionelle Übertragung der Nominativstellenqualität der jeweils zweiten, monovalenten Verbverwendungen auf es: Das es wird, wobei das Allomorph es ...-t weiterhin Träger von ,Singular' und ,3. Person' bleibt, zu einer Nominativergänzung in Rede. ${ }^{33}$

Dabei wird auch deutlich, warum diese Art von Kontraststellung von „normbewußten“ Sprachteilhabern und Linguisten in Frage gestellt wird: Erstens, weil hier jeweils zwei verschiedene Verbvarianten kontrastiert werden. Zweitens, weil dementsprechend die Verbformen, die in der Reduktion geopfert wurden, zugunsten von nur quasi stellvertretenden Verbformen geopfert wurden, denn diese können die verbalen Nominativkategorien der nichtrealisierten Verbformen nicht tragen. Somit konnte dem erwähnten „Unbehagen der Muttersprachler" eine modellinterne Erklärung zugewiesen werden.

Es ist eine andere, nicht unmittelbar zu unserem Thema gehörende Frage, wozu das heutige Deutsch dieses es, das sogar „das Verstehen behindern“ kann (Heringer (1988), 86), überhaupt noch braucht. ${ }^{34}$ Obwohl man die Frage in dieser ahistorischen Form gar nicht stellen dürfte, ist die Vermutung sicherlich nicht verfehlt, daß diese Art Zweigliedrigkeit im Althochdeutschen deshalb (!) auftauchte (und mit dem Ende der mittelhochdeutschen Periode im wesentlichen obligatorisch wurde) ${ }^{35}$ weil(!) ${ }^{36}$ das Personalpronomen für die schwindende Anaphorizität/Deiktizität des Verbflexivs einspringen mußte, wodurch die zweigliedrige (genauer: die zwei Stellungsglieder enthaltende) Satzform zur Standardform des finiten Aussagesatzes wurde. ${ }^{37}$ (Dabei drückte aber das es impersonale von vornherein nur die Kategorien ,Singular' und ,3. Person' aus, diente also keiner Erstaktantenbezeichnung.) Parallel zu dieser Entwicklung - oder vielleicht als deren funktionale Begründung - kommt es zur Grammatikalisierung der thematischen Progression in dem Sinne, daß der finite Aussagesatz über eine Thema-Konstituente verfügen muß.

Eine synchrone Erklärung ergibt sich sowohl aus unserer Skizze der getrennten Kategorienrealisierungen (vgl. 1.3.5.2.) wie auch aus den Paradigmenkategorien der unpersönlichen Verben:

${ }^{33}$ Einer Nominativergänzung also, die nicht durch paradigmatische Aktualisierung der eigenen E 1-Stelle, sondern durch syntagmatische Übertragung einer anderen „entstanden" ist.

${ }^{34}$ Es kommen u. a. topologische Gründe (Es regnet. vs. Regnet es?) in Frage, man sollte jedoch nicht übersehen, daß bereits die Intoneme funktional sind.

35 Vgl. Heringer (1967), 34 und Lenerz (1985), $107 \mathrm{f}$.

${ }^{36}$ Deshalb und weil sind nicht im Sinne einer kausalistischen Sprachwandelauffassung zu verstehen.

37 Nach Buscha (1988), 31 hat das ,formale Subjekt ES“ die Funktion, „die Zweigliedrigkeit als Minimalstruktur des deutschen Satzes zu sichern“. 
Einerseits geht von der nominalen Kategorie ,3. Person' ein Druck auf die nominale Realisierung der Personenkategorie aus. Strukturell wird „etwas“, das wegen der fehlenden funktionalen Nominativrealisierung kein Makroaktant sein kann, das aber an der Realisierung der Kategorien ,3. Person' und ,Singular' beteiligt sein muß, erwartet. Andererseits fordern die Paradigmenkategorien ,3. Person' und ,Singular' des Verbs die Realisierung der nominalen Konstituentenkategorien ,3. Person' und ,Singular', damit sich die Rektionsbeziehungen hinsichtlich Person und Numerus einstellen können. Da von den sog. Personalpronomina (3. Person) des Deutschen nur es genusneutral realisiert werden kann, kann nur es der genannten „etwas"-Funktion gerecht werden: In es schneit und dergl. ist es Bestandteil des Allomorphs des Morphems ,Singular 3. Person', also von es ...-t.

\section{Subjekt und das Zwei-Ebenen-Modell}

In Darstellungen, in denen die Frage der Sonderstellung des Subjekts diskutiert wird, wird nicht immer geklärt, ob unter ,Sonderstellung' sein Status im Vergleich zu anderen nominalen Satzgliedern oder im Rahmen einer grundlegenden Zweiteilung des Satzes in Subjekt und Prädikat $\mathrm{zu}$ verstehen ist. ${ }^{38}$ Diese beiden „Sonderstellungen“ beziehen sich auf völlig verschiedene Ebenen: die erste auf die Beschreibung einer Einzelsprache, die andere auf die methodologischen Grundlagen einer Theorie.

In einem strukturell-typologisch fundierten Valenzmodell, das sich des Zwei-Ebenen-Modells bedient, ist die zweite Art Sonderstellung des Subjekts von vornherein ausgeschlossen, da sie (a) dem Unterschied zwischen Sprachen

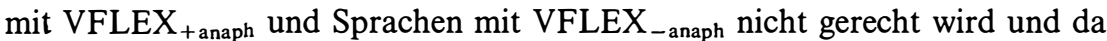
(b) in zahlreichen Sprachen die (strukturelle) mikrovalenzielle Realisierung auch von Zweit- und Drittaktanten möglich ist (vgl. Anm. 14). Darüber hinaus gibt es den Sprachtyp mit mikrovalenzieller Realisierung des Zweit- und Drittaktanten - jedoch ohne die des Erstaktanten (Beispiel: Indonesisch, vgl. Pasierbsky (1981), 174f.). Die Annahme einer universellen Zweiteilung des Satzes ergäbe daher keinen Sinn, vielmehr müßte man, wollte man die zentrale Stellung des verbalen Valenzträgers nicht anerkennen, je nach Sprachtyp unterschiedliche Zwei-, Drei- und Vierteilungen vornehmen.

Gegen die erste Art Sonderstellung, die ja per definitionem einzelsprachbezogen ist, hat der Valenzforscher im Prinzip nichts einzuwenden. Und dies nicht nur deshalb, weil es offenkundig ist, daß Erstaktanten bevorzugte Träger

38 Sauber unterscheidet zwischen diesen beiden Interpretationen Järventausta (1991, 58 ff.), nicht jedoch Wegener $(1990,153)$ : Mit ihrem Kongruenzargument plädiert sie für die Zweiteilung des Satzes, mit ihrem zweiten Argument („Das Subjekt hat wenig psychische Realität im Vergleich zu den Objekten, wie Tests [...] und wie auch die Zitierformen [jmdm. etw. stehlen, etw. aus etw. schließen, auf jmdn. warten - V. Á.] beweisen, die wir assoziieren, wenn wir einen Infinitiv hören [...].") für das Subjekt als Primus inter pares. 
bestimmter Qualitäten sein können (vgl. z. B. Keenan (1976), 312 ff.; für Ergänzungsklassen des Deutschen vgl. Askedal (1988), insb. 28), sondern auch deshalb, weil er sich hier gern zu den Grenzen des Kompetenzbereiches seiner Theorie bekennt. Ob eine Beschreibungskategorie wie ,Subjekt' für die untersuchte Einzelsprache relevant ist, läßt sich jedoch nicht voraussagen, sondern es muß im Sinne von Reis (1982) von Sprache zu Sprache neu überprüft werden. Sollte die Relevanz einer Beschreibungskategorie wie ,Subjekt' für eine Sprache $L_{1}$ erwiesen sein, was nach Reis für das Deutsche nicht zutrifft, kann die Untersuchung der Subjekteigenschaften wohl kaum zum Gegenstand eines strukturell-typologisch orientierten Valenzansatzes gemacht werden. Vielmehr muß sie im Rahmen einer allgemeinen Grammatiktheorie angestellt werden. Dabei ist ,allgemein' nicht nur im Sinne von ,universell, die signifikanten Eigenschaften von Einzelsprachen untersuchend', sondern auch im Sinne von, alle Gebiete einer Einzelsprache bzw. überhaupt von sprachlichen Aktivitäten (und Passivitäten) umfassend' zu verstehen. Nur im Rahmen einer solchen Theorie kann entschieden werden, ob eine bestimmte Eigenschaft, die in der Sprache $L_{1}$ materiell am Erstaktanten realisiert wird bzw. ihm zugeschrieben werden kann, oder ein bestimmter Prozeß, an dem der Erstaktant mitwirkt, dem Erstaktanten in jener Sprache den Status eines Subjekts verleihen oder nicht. Für eine VP-intern generierte Subjektposition im Deutschen argumentiert Haider (1984).

\section{Kongruenz, Rektion und das Zwei-Ebenen-Modell}

4.1. Im Rahmen eines funktionalen Ansatzes stellt eine Kongruenzverletzung wie *Dies sind ein Platz nicht einfach das ungrammatische Pendant zu grammatischem Dies ist ein Platz dar, sondern der wesentliche Unterschied zwischen beiden Sätzen besteht darin, daß der erste Satz deshalb ungrammatisch ist, weil funktionale Kategorien wie Singular und Plural kraft ihrer einzelsprachlichen Opposition einzelsprachliche Inhalte, also (grammatische) Bedeutungen transportieren (Coseriu (1987 a), 177f.). Die zahlreichen Kongruenzunsicherheiten im Deutschen (z. B. 100 Stück pro Tag sind/ist das Ziel), die scheinbaren Ausnahmen des Englischen (The police are ...) oder eben die Koordinationen (Maria und Peter schlafen) sind (für mich) intuitiv und im gleichen Erklärungszusammenhang zu beschreiben, wenn man zumindest die funktionalen Teile der Grammatik als semantisch auffaßt. Für den kommunizierenden Sprachteilhaber stellen ja „Fehler“, d. i. grammatische Inkorrektheiten, zumeist semantische Interpretationsprobleme oder eben -aufgaben dar. Auch im Falle von *Dies sind ein Platz nimmt „unsere Intuition“ unmittelbar nicht an der Kongruenzverletzung Anstoß, sondern wir haben Schwierigkeiten, Dies sind zu ein Platz semantisch in Beziehung zu setzen. Der Fragenkomplex ist analog zu dem in Anm. 28 angeschnittenen Passivproblem.

4.2. Personen- und Numeruskongruenzen sind unabhängig vom Sprachtyp und ganz im Sinne der traditionellen Analyse zwischen Verbform und makro- 
valenzieller Realisierungsebene anzunehmen. Die normgemäße Erscheinungsform funktionaler Personen- und Numeruskategorien ist in Sprachen mit VFLEX $_{+ \text {anaph }}$ mikrovalenziell, in denen mit VFLEX - anaph $_{\text {mikro- und makro- }}$ valenziell. Dabei impliziert die Einschränkung normgemäß, daß es durchaus möglich ist, besondere Diskurseffekte dadurch zu erzielen, daß man Kongruenzbeziehungen verletzt, daß man also Mikro- und Makroebene durch die Realisierung unterschiedlicher funktionaler Kategorien gegeneinander ausspielt. Besonders (oder ausschließlich?) geeignet sind für solche Diskurseffekte Sprachen mit VFLEX $+_{\text {anaph }}$, denn bei diesen brauchen die VFLEX nicht extra, durch besondere Kontexttricks anaphorisiert zu werden, sondern es muß nur verhindert werden, da $\beta$ die Makroebene die natürliche anaphorische Potenz von VFLEX „verschluckt“. Es muß sozusagen ein „Diskurskeil“ zwischen beide Ebenen getrieben werden, um die Komplementarität der Makro-/Mikrorealisierung okkasionell aufzuheben, oder andersherum: um der mikrovalenziellen Repräsentation trotz makrovalenzieller Realisierung okkasionell den Rang einer mikrovalenziellen Realisierung zu verleihen.

Ein Beispiel aus dem Ungarischen: Freunde speisen in einem Lokal. Als es zum Zahlen kommt, stellt einer von ihnen die gewohnte Frage: Wer ist heute dran? Die anderen antworten im Chor: Te fizetünk (,Du zahlen [wir] oder ,Du zahl-wir'). Auf diese Weise geben sie dem Unglücksraben zwar klar zu verstehen, daß er für die ganze Zeche aufzukommen hat. Sie tun jedoch scherzhaft - durch das Pluralsuffix - so, als ob eine anteilige Zahlungsabsicht vorliegt. Als Antwort in der obigen Situation wäre übrigens auch Mi fizetsz (,Wir zahlst' oder, Wir zahl-du') denkbar.

Angesichts der zahlreichen Möglichkeiten, Diskurseffekte durch bewußte Verletzung von verschiedenen syntagmatischen Relationen zu erzielen, stellt sich übrigens auch die Frage, wie sich diese Effekte am INFL-Knoten der GB adäquat darstellen lassen. Denn in solchen Fällen wird in der Performanz nicht einfach - sozusagen zusätzlich - von der Kompetenz abgewichen, sondern der Sprecher manipuliert an der eigenen Kompetenz. Die Abweichung muß also in der strukturellen Ableitung des (bewußt ungrammatisch gestalteten) Sprachproduktionsprozesses sichtbar gemacht werden können.

Was die in 1.2. diskutierten Inkorrektheiten $\left({ }^{*}\right.$ Mich $\left(/{ }^{*}\right.$ Meiner $/{ }^{*}$ Mir $)$ gehe usw.) anbelangt, stellen sie - wie erwähnt - Verletzungen einer Rektionsbeziehung dar. Wie „sehr empirisch“ Rektionsbeziehungen sind, wird daran deutlich, daß ein Hörer, dessen Gesprächspartner seinen Turn etwa mit Mir gehe ... anfängt, zuerst keinen Fehler wittern, sondern wohlwollend und in Anlehnung an eine Variante desselben Verbs versuchen wird, ,Dativ' als eine Paradigmenkategorie von geh- zu interpretieren.

Abschließend sollte vielleicht noch klargestellt werden, daß der Umstand, $\mathrm{da} ß$ nichtpronominale (substantivische) Makro-E 1en die realisierte Verbform hinsichtlich der Kategorie ,3. Person' regieren (vgl. 1.2.), den Dependens-Status der E1 nicht antastet. Wie in 1.4.1. festgestellt wurde, gehören Person und Numerus nicht zur Valenzpotenz des Verbs. Der Dependens-Status der E 1 basiert auf der Realisierung der Nominativkategorie. 
4.3. Die integrative Betrachtung der Realisierung der E1 auf Mikro- und Makroebene liefert ein wichtiges Argument gegen die Nichtunterscheidbarkeit von E 1 und sog. Prädikatsnomen (zum Problem vgl. Eisenberg (1989), 288 ff.; zum Status der Nominalverben vgl. Ágel (1989)). Selbst in einem Satz wie Kinder bleiben Kinder kann von keiner strukturellen Gleichstellung der beiden Nominalphrasen gesprochen werden, denn an den VFLEX des Deutschen kann nur eine potentielle Mikroebene realisiert werden: die der E1. Egal, welche Nominalphrase man für die Makro-E 1 hält, eine von beiden ist sie.

\section{Ellipsen und das Zwei-Ebenen-Modell}

Es kann nicht die Aufgabe dieser Arbeit sein, sich an der schwierigen Ellipsendiskussion zu beteiligen, hier kann es nur darum gehen, bestimmte Zusammenhänge zwischen dem Zwei-Ebenen-Modell und einigen Ellipsenproblemen herzustellen. Was den ellipsentheoretischen Hintergrund anbelangt, schließe ich mich Wolfgang Kleins Auffassung an, (auch) Ellipsen seien strukturelle „Nahtstellen“, an denen natürliche Sprachen ,direkt auf die Integration des Beiwissens ausgelegt sind" $(1984,131)$. Mit Nachdruck zu betonen sei außerdem, daß Ellipsen - nomen non est in jeder Hinsicht omen - vollkommen normgemäße Konstruktionen darstellen, weshalb im Diskurs vielfach gerade die 1:1-Realisierung einer Grundstruktur - statt der erwartbaren Aktualisierung einer Ellipse - als unangemessen anzusehen ist. ${ }^{39}$

Für die Zwecke der anschließenden Erörterungen reicht eine Grobeinteilung der Ellipsen in grammatische oder Kontextellipsen (hier am Beispiel der Koordinationsellipsen) und lexikalisch-pragmatische Ellipsen (hier nur: Textsorten- und Topikellipsen, vgl. Klein (1984), $118 \mathrm{ff}$. und Schwitalla (1988)). Zum Status von indefiniten Auslassungen im Zwei-Ebenen-Modell sei auf 1.3.7. verwiesen.

5.1. Der Standpunkt des Primats der Mikroebene wird erhärtet durch Untersuchungen über Koordinationsellipsen (fürs Deutsche vgl. z. B. Klein (1984), 134 ff. und Müller (1990), 352 f.). Während nämlich die Ersparung syntaktisch realisierter Satzglieder oder Gliedteile zur heutigen deutschen Sprachnormalität gehört (er lebt und arbeitet in Heidelberg; zahmes und wildes Schwein), ist die „Ellipse bei gleichen Flexionsendungen“ (Müller, ebd.) im heutigen Deutsch zwar natürlich möglich, aber keinesfalls normgemäß. Der Verfechter des ZweiEbenen-Modells braucht sich aber nicht in Normdiskussionen zu verwickeln, denn es gibt hier einen entscheidenden Unterschied zwischen dem nominalen und dem verbalen Bereich: Während Koordinationsellipsen im Bereich der Adjektivflexion (Karl und Rosas Ideale in der DDR verwirklicht [Zeitungstitel];

39 Wie in Anm. 25 angedeutet, wird hier eine „autonomistische Position“ eingenommen. Zum Vergleich der beiden Grundpositionen ,autonomistisch' und ,reduktionistisch " vgl. etwa Dentler (1990), $12 \mathrm{ff}$. 
oder aus früheren Zeiten: zahm- und wildes Schwein [Uhland] - Belege in Müller, ebd.) zweifelsohne systemgerecht sind, ist diese Art Reduktion im verbalen Bereich nicht nur nicht normgemäß, sondern m. E. auch nicht systemgerecht: *er leb- und arbeitet in Heidelberg. Die Frage ist, ob man mit Hilfe des Zwei-Ebenen-Modells für die Nicht-Systemgerechtheit argumentieren und so ein Kriterium für verbale Koordinationsellipsen finden könnte. Man betrachte die folgenden, m. E. abnehmende Angemessenheitsstufen vertretenden Beispiele:

[1] er lebt und arbeitet in Heidelberg

[2] (*)lebt- und arbeitet in Heidelberg

[3] *er leb- und arbeitet in Heidelberg

[4] *leb- und arbeitet in Heidelberg

Die beiden Problemfälle liegen zwischen korrektem [1] und deutlich systemwidrigem [4]. Der entscheidende Unterschied zwischen [2], den man sich etwa in Texten der gesprochenen Umgangssprache vorstellen kann, und [3] besteht darin, daß in [2] sowohl die E 1 von leb-als auch die von arbeit- auf der Mikroebene realisiert sind. Die restlose mikrovalenzielle Realisierung scheint also mehr ins Gewicht zu fallen als die Realisierung des Personalpronomens, obwohl letzteres, im Gegensatz zu $-t$, kategoriell eindeutig ist. ${ }^{40}$ Dies ist im Lichte des in 1.4.2. Gesagten nicht verwunderlich, denn man kann sich schlecht Diskurssituationen vorstellen, die in einer Koordination in dem Sinne einen Druck auf die Realisierungen ausüben, daß das erste Koordinationsglied nur makrovalenziell, das zweite nur mikrovalenziell zu realisieren ist. Damit haben wir ein gewichtiges Argument für den Primat der Mikroebene und das Zwei-Ebenen-Modell gewonnen. Noch wichtiger erscheint mir jedoch, daß sich hier eine Minimalbedingung für Reduktionen des Deutschen - vielleicht auch anderer Sprachen - abzeichnet: Reduktionen im Bereich des Verbs dürfen die mikrovalenzielle Realisierung von Aktanten nicht antasten.

5.2. Lexikalisch-pragmatische Ellipsen sind in dem Sinne immer nur als nichteinleitende Teile von Diskursen denkbar, als sie sich auf Situationswissen und/ oder Kontextinformation stützen müssen. ${ }^{41}$ Deutliche Belege sind Mach ich oder Gibt es (Schwitalla (1985), 267). Die oben formulierte Minimalbedingung gilt auch für sie, wie die folgenden Beispiele bezeugen:

${ }^{40}$ Das gleiche gilt für das Ungarische. Keiner der Mikroaktanten kann in Koordinationsellipsen aufgehoben werden. Psycholinguistisch gesehen gründet die Nichtakzeptabilität von [3] wohl in erster Linie auf einer zu hohen Verarbeitungserwartung gegenüber dem Hörer. Gleichzeitig und im gleichen komplexen Nukleus werden eine Interpretation vom identischen Anfangsstück her, d. h. eine „Vorwärtsellipse“ ( $e r$... [er] arbeitet) und eine vom identischen Endstück her, d. h. eine „Rückwärtsellipse“ (leb-[t] ... arbeitet) vorausgesetzt.

41 In Klein (1984), $130 \mathrm{f}$. werden neben „Weltwissen“ diese zwei Komponenten des Beiwissens genannt. 
Beim klassischen Beispiel der Aufhebung, ${ }^{42}$ der Textsortenellipse ankomme morgen (vgl. auch frz. arrivons demain in 1.3.4.) übernimmt die Mikroebene die Bezeichnung des Erstaktanten. Ähnlich markiert in der Topik-Ellipse riecht gut das „kommunikative“ Deiktikon - $t$ eine Aufhebungsform, die unter der speziellen kommunikativen Bedingung eines Sprecher und Hörer gemeinsamen Wahrnehmungshorizontes (vgl. Schwitalla (1988), 76) zustande gekommen ist. Die syntagmatischen Relationen zwischen ich und ankomme bzw. es und riecht weichen einer Art „kommunikativer Kongruenz“ zwischen „Realität“ (Schreiber bzw. Wahrnehmungsgröße) und Sprechen (ankomme bzw. riecht).

\section{Standardsprache und das Zwei-Ebenen-Modell}

Gemeinsam dem Zwei-Ebenen-Modell und dem pro-drop-Parameter ist die stillschweigende Annahme ausgezeichneter einzelsprachlicher Varietäten, die sozusagen den strukturellen Normalfall darstellen. Diese Varietäten sind zum Großteil Standardsprachen bzw. Standardvarietäten, in einigen wenigen Fällen auch Dialekte. Was als Struktur und was als Modifikation einer Struktur gilt, widerspiegelt daher u. a. unsere gewissermaßen normative Wertordnung funktioneller Sprachen. Wir sprechen zwar vom „Deutschen“, meinen jedoch nicht die historische Sprache ,Deutsch“ in ihrer ganzen Komplexität (,historische Sprache' im Sinne Coserius), sondern nur eine von den zahlreichen funktionellen Sprachen, die diese historische Sprache ausmachen: die Standardsprache (oder genauer: die bundesdeutsche Standardvarietät der Standardsprache). Soziolekte und Stile werden in die Bildung struktureller Modelle höchstens nur als Kandidaten für Modifikationen mit einbezogen.

Die Arbeit mit dieser Wertordnung - ob expliziert oder nicht - ist in der strukturellen Linguistik nichts Überraschendes. Oft ist sie sogar notwendig. Nichtsdestoweniger kann als ein möglicher Adäquatheitsmaßstab struktureller Modelle ihre Integrierbarkeit in soziolinguistische Modelle angesehen werden (0.2.). Sind sie flexibel genug, um integriert werden zu können?

Die Integrierbarkeit des Zwei-Ebenen-Modells scheint mir gewährleistet. Hier wurden Modifikationen etwa der deutschen Valenzrealisierungsstruktur als diskursbedingte Gleichgewichtsverluste beschrieben, die „ungarische/italienische Normalzustände" hervorrufen. Wenn also zum Zweck einer soziolinguistischen Untersuchung eine funktionelle Sprache des Deutschen isoliert werden soll, für die durchweg solche „ungarischen/italienischen“ Strukturen charakteristisch sind, können die im Vergleich zur Standardstruktur als Modifikationen beschriebenen Realisierungsstrukturen als die normalen Realisierungsstrukturen dieser funktionellen Sprache redefiniert werden. Aus dieser soziolinguistischen Sicht besteht demnach die historische Sprache ,Deutsch“

42 Gute Beispiele gibt es u. a. in Reis (1982), 190. Diese sind allerdings unter der engen Bezeichnung ,Textsortenellipse' nicht zu erfassen, es geht um Beispiele aus der gesprochenen Umgangssprache. Vgl. auch Bossong (1980), 14. 
aus funktionellen Sprachen mit Realisierungsstrukturen, die ansonsten verschiedenen Einzelsprachen zugeschrieben werden. Deutsch ist dann keine Spra-

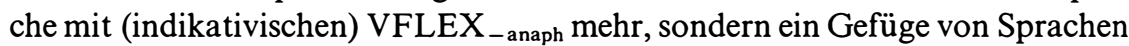
mit VFLEX $_{+_{\text {anaph }}}$ und VFLEX - anaph und ihren möglichen Übergängen. Als Modifikationen bleiben nur diejenigen Strukturen zu beschreiben, die unabhängig von funktionalsprachlichen Kriterien produziert werden.

\section{Schlußbemerkungen}

Im Anschluß an Heringer (1984) resümiert Helbig $(1991,58)$ den aktuellen Forschungsstand wie folgt: „Die Zentralität des Verbs resultiert [...] nicht mehr - wie bei der syntaktisch orientierten Valenztheorie - aus der Satzbildungspotenz, sondern aus der Szenen- oder genauer Perspektivenbildungspotenz des Verbs. Ob diese Akzentverlagerung [...] tatsächlich als ,Umkehr' (und damit als undialektischer Ersatz des alten Wertsystems durch ein neues) verstanden werden darf, bedarf indes der genaueren Prüfung."

Ich denke, das Zwei-Ebenen-Modell kann als ein Beitrag zu dieser „genaueren Prüfung" aufgefaßt werden. Denn es kann nicht darum gehen, das alte Wertsystem „undialektisch“" wegzuwerfen, sondern nur darum zu prüfen, wie die Satzbildungspotenz eine moderne Interpretation, die mit Erkenntnissen über die Szenenbildungspotenz des Verbs konform ist, erfahren kann.

Es ist nicht abzusehen, ob sich die germanistische Valenzforschung mit diesem Modell anfreunden wird. Dafür könnten allerdings über die in den Abschnitten 2-6 angedeuteten Anwendungsmöglichkeiten hinaus sprechen:

(a) Die Lösbarkeit des valenziellen Status des deutschen Imperativs;

(b) Die Möglichkeit, verschiedene Diskursphänomene nicht nur als „Valenzverletzungen" auszuweisen, sondern auch ihre Effekte strukturell zu interpretieren;

(c) Die vielen - insbesondere an/mit dem IdS - laufenden kontrastiven Valenzprojekte (vgl. Schumacher (1988)).

$\mathrm{Da}$ ein eventueller Erfolg des Zwei-Ebenen-Modells auch wichtige Umstellungen im schulischen Grammatikunterricht insgesamt erfordern würde (man denke nur an die Notwendigkeit der Uminterpretation der traditionellen Fragemethode für Satzglieder), braucht freilich den Grammatiker von heute nicht zu beunruhigen.

\section{Literatur}

Abraham, Werner (Hg.) (1982): Satzglieder im Deutschen. Vorschläge zur syntaktischen, semantischen und pragmatischen Fundierung (Studien zur deutschen Grammatik 15). Tübingen.

Abraham, Werner (Hg.) (1985): Erklärende Syntax des Deutschen (Studien zur deutschen Grammatik 25). Tübingen.

Abraham, Werner/Kosmeijer, Wim/Reuland, Eric (Hg.) (1991): Issues in Germanic Syntax (Trends in Linguistics; Studies and Monographs 44). Berlin et al.

Agazzi, Evandro (Hg.) (1990): La comparabilité des théories scientifiques/Die Vergleichbarkeit wissenschaftlicher Theorien. Fribourg/Freiburg. 
Ágel, Vilmos (1988): Überlegungen zur Theorie und Methode der historisch-synchronen Valenzsyntax und Valenzlexikographie. Mit einem Verbvalenzlexikon zu den Denkwürdigkeiten der Helene Kottannerin (1439-1440) (Lexicographica: Series Maior 25). Tübingen.

Ágel, Vilmos (1989): Zum Status der Nominalverben in der Valenztheorie. In: Germanistisches Jahrbuch DDR - Ungarn 8, 210-219.

Ágel, Vilmos (1991): Nochmals: Redundanz. In: Bartha/Brdar Szabó (Hg.) (1991) $61-74$.

Andrews, Avery (1985): The major functions of the noun phrase. In: Shopen (Hg.) (1985) 62-154.

Askedal, John Ole (1988): Sprachliche Valenz unter dem Aspekt der Arbitrarität und Konventionalisierung. In: Helbig (Hg.) (1988) 22-36.

Askedal, John Ole (1990): Zur syntaktischen und referentiell-semantischen Typisierung der deutschen Pronominalform es. In: DaF 27, 213-225.

Bartha, Magda/Brdar Szabó, Rita (Hg.) (1991): Von der Schulgrammatik zur Allgemeinen Sprachwissenschaft, Beiträge zur Gedenktagung für Professor János Juhász (Budapester Beiträge zur Germanistik 23). Budapest.

Bloomfield, Leonard (1933): Language. New York.

Boas, Franz (1911): Introduction to the Handbook of American Indian Languages. Washington.

Bossong, Georg (1980): Aktantenfunktionen im romanischen Verbalsystem. In: Zeitschrift für romanische Philologie 96, 1-22.

Buscha, Joachim (1988): Die Funktionen der Pronominalform ES. In: DaF 25, 27-33.

Bühler, Karl (1934): Sprachtheorie. Die Darstellungsfunktion der Sprache (UTB 1159). Stuttgart et al. 1982 [ungekürzter Neudruck der Ausgabe von 1934].

Bynon, Theodora (1990): Pronoun and verb agreement in typological and diachronic perspective. In: SOAS Working Papers in Linguistics 1, 97-111.

Chomsky, Noam (1986): Knowledge of Language: Its Nature, Origin, and Use (Convergence). New York.

Comrie, Bernard (1989): Language Universals and Linguistic Typology. Syntax and Morphology. Second Edition. Oxford.

Coseriu, Eugenio (1974): Synchronie, Diachronie und Geschichte. Das Problem des Sprachwandels (Internationale Bibliothek für Allgemeine Linguistik 3). München.

Coseriu, Eugenio (1987): Formen und Funktionen. Studien zur Grammatik (Konzepte der Sprach- und Literaturwissenschaft 33). Tübingen.

Coseriu, Eugenio (1987a): Bedeutung, Bezeichnung und sprachliche Kategorien. In: Coseriu (1987) 177-198.

Coseriu, Eugenio (1988): Sprachkompetenz. Grundzüge der Theorie des Sprechens (UTB 1481). Tübingen.

Dentler, Sigrid (1990): Verb und Ellipse im heutigen Deutsch. Zum „Fehlen“ verbabhängiger Bestimmungen in Theorie und Praxis (Göteborger Germanistische Forschungen 31). Göteborg.

Eisenberg, Peter (1989): Grundriß der deutschen Grammatik. 2., überarb. und erw. Aufl. Stuttgart.

Emons, Rudolf (1978): Valenzgrammatik für das Englische. Eine Einführung (Anglistische Arbeitshefte 16). Tübingen.

Engel, Ulrich (1982): Syntax der deutschen Gegenwartssprache (Grundlagen der Germanistik 22). 2., überarb. Auflage. Berlin.

Engelen, Bernhard (1984): Einführung in die Syntax der deutschen Sprache. Bd. I: Vorfragen und Grundlagen. Baltmannsweiler.

Engelen, Bernhard (1986): Einführung in die Syntax der deutschen Sprache. Bd. II: Satzglieder und Satzbaupläne. Baltmannsweiler.

Erben, Johannes (1980): Deutsche Grammatik. Ein Abriß. 11., völlig neu bearb. Aufl. von „Abriß der deutschen Grammatik“. 12. Aufl. München. 
Eroms, Hans-Werner (1985): Eine reine Dependenzgrammatik für das Deutsche. In: Deutsche Sprache 13, 306-326.

Fanselow, Gisbert/Felix, Sascha W. (1990): Sprachtheorie. Eine Einführung in die Generative Grammatik. Band 1: Grundlagen und Zielsetzungen (UTB 1441). 2. Aufl. Tübingen.

Fanselow, Gisbert/Felix, Sascha W. (1990a): Sprachtheorie. Eine Einführung in die Generative Grammatik. Band 2: Die Rektions- und Bindungstheorie (UTB 1442). 2., durchges. Aufl. Tübingen.

Festschrift Mollay (1978): Festschrift Karl Mollay zum 65. Geburtstag (Budapester Beiträge zur Germanistik 4). Budapest.

Fourquet, Jean (1970): Prolegomena zu einer deutschen Grammatik (Sprache der Gegenwart 7). Düsseldorf.

Franks, Steven (1990): On the status of null expletives. In: Lingua 81, 1-24.

Givon, Talmy (1984): Syntax: a functional-typological introduction. Bd. 1. Amsterdam et al.

Graumann, Carl Friedrich/Hermann, Theo (Hg.) (1984): Karl Bühlers Axiomatik: 50 Jahre Axiomatik der Sprachwissenschaft. Frankfurt am Main.

Grewendorf, Günther (1991): Aspekte der deutschen Syntax. Eine Rektions-BindungsAnalyse (Studien zur deutschen Grammatik 33). 2. Aufl. Tübingen.

Grewendorf, Günther/Hamm, Fritz/Sternefeld, Wolfgang (1987): Sprachliches Wissen. Eine Einführung in moderne Theorien der grammatischen Beschreibung (Suhrkamp-Taschenbuch Wissenschaft 695). Frankfurt am Main.

Haider, Hubert (1984): Topik, Focus \& V-Second. In: Groninger Arbeiten zur Generativen Linguistik $21,72-120$.

Haider, Hubert (1991): Null subjects and expletives in Romance and Germanic languages. In: Abraham/Kosmeijer/Reuland (Hg.) (1991) 49-66.

Heger, Klaus (1991): Redundanz in der Morphologie des Deutschen? In: Bartha/Brdar Szabó (Hg.) (1991) 53-59.

Helbig, Gerhard (1988): Zum Verhältnis von Grammatik und Lexikon (aus der Sicht der Sprachwissenschaft und des Fremdsprachenunterrichts). In: DaF 25, 160-167.

Helbig, Gerhard (Hg.) (1988): Valenz, semantische Kasus und/oder „Szenen“ (LS/ZISW/ A 180). Berlin.

Helbig, Gerhard (1991): Entwicklungen und Kontroversen in der Valenztheorie. In: Iwasaki/Shichiji (Hg.) (1991) 44-61.

Heringer, Hans Jürgen (1967): Wertigkeiten und nullwertige Verben im Deutschen. In: Zeitschrift für deutsche Sprache 23, 13-34.

Heringer, Hans Jürgen (1970): Theorie der deutschen Syntax (Linguistische Reihe 1). München.

Heringer, Hans Jürgen (1984): Neues von der Verbszene. In: Stickel (Hg.) (1984) 34-64.

Heringer, Hans Jürgen (1985): The Verb and its Semantic Power: Association as a Basis for Valency Theory. In: Journal of Semantics 4, 79-99.

Heringer, Hans Jürgen (1988): Lesen lehren lernen: Eine rezeptive Grammatik des Deutschen. Tübingen.

Heringer, Hans Jürgen/Strecker, Bruno/Wimmer, Rainer (1980): Syntax. Fragen Lösungen - Alternativen (UTB 251). München.

Hermon, Gabriella/Yoon, James (1989): The Licensing and Identification of pro and the Typology of AGR. In: CLS 25, 174-192.

Huang, C.-T. James (1984): On the Distribution and Reference of Empty Pronouns. In: Linguistic Inquiry 15, $531-574$.

Huang, C.-T. James (1989): Pro-Drop in Chinese: A Generalized Control Theory. In: Jaeggli/Safir (Hg.) (1989) 185-214.

Iwasaki, Eijirō/Shichiji, Yoshinori (Hg.) (1991): Internationaler Germanisten-Kongreß in Tokyo. Ansprachen, Plenarvorträge, Berichte. München. 
Jaeggli, Osvaldo/Safir, Kenneth J. (1989): The Null Subject Parameter and Parametric Theory. In: Dieselben (Hg.) (1989) $1-44$.

Jaeggli, Osvaldo/Safir, Kenneth J. (Hg.) (1989): The Null Subject Parameter (Studies in Natural Language and Linguistic Theory 15). Dordrecht et al.

Järventausta, Marja (1991): Das Subjekt im Deutschen und im Finnischen (Werkstattreihe Deutsch als Fremdsprache 30). Frankfurt am Main et al.

Keenan, Edward L. (1976): Towards a Universal Definition of „Subject“. In: Li (Hg.) (1976) 303-333.

Keller, Rudi (1990): Sprachwandel (UTB 1567). Tübingen.

Kenesi, István (Hg.) (1987): Approaches to Hungarian II: Theories and Analyses. Szeged.

É. Kiss, Katalin (1987): Is the VP universal? In: Kenesei (Hg.) (1987) 13-85.

Klein, Wolfgang (1984): Bühler Ellipse. In: Graumann/Hermann (Hg.) (1984) 117-141.

Kuhn, Thomas S. (1976): Die Struktur wissenschaftlicher Revolutionen. 2., revidierte und um das Postskriptum von 1969 ergänzte Aufl. (Suhrkamp-Taschenbuch Wissenschaft 25). Frankfurt am Main. [engl. Orig.: The Structure of Scientific Revolutions 2. ed. 1970]

László, Sarolta (1978): Einige Überlegungen zu einer vergleichenden Valenzanalyse ungarischer und deutscher Verben. In: Festschrift Mollay (1978) 159-169.

László, Sarolta (1988): Mikroebene. In: Mrazović/Teubert (Hg.) (1988) 218-233.

Lehmann, Christian (1982): Universal and typological aspects of agreement. In: Seiler/ Stachowiak (Hg.) (1982) $201-267$.

Lenerz, Jürgen (1985): Zur Theorie syntaktischen Wandels. Das expletive es in der Geschichte des Deutschen. In: Abraham (Hg.) (1985) 99-136.

Li, Charles N. (Hg.) (1976): Subject and Topic. New York et al.

Lyons, John (1971): Einführung in die moderne Linguistik. München. [engl. Orig.: Introduction to Theoretical Linguistics 1968]

Martinet, André (1980): Eléments de linguistique générale. Nouvelle édition remaniée et mise à jour 1980 [1. Aufl. 1970]. Paris.

Matthews, Peter H. (1981): Syntax (Cambridge textbooks in linguistics). Cambridge.

Mosel, Ulrike (1984): Towards a typology of valency. In: Arbeiten des Kölner Universalienprojekts, Institut für Sprachwissenschaft 58, 1-19.

Mrazović, Pavica/Teubert, Wolfgang (Hg.) (1988): Valenzen im Kontrast. Ulrich Engel zum 60. Geburtstag. Heidelberg.

Mudersbach, Klaus (1983): Hol-Atomismus als Vereinheitlichung von Atomismus und Holismus. In: Weingartner/Czermak (Hg.) (1983) 347-349.

Mudersbach, Klaus (1990): Theorien-Vergleich und Vereinheitlichung von Atomismus und Holismus. In: Agazzi (Hg.) (1990) 87-95.

Müller, Gereon/Rohrbacher, Bernhard (1989): Eine Geschichte ohne Subjekt. Zur Entwicklung der pro-Theorie. In: Linguistische Berichte Heft 119, 3-52.

Müller, Wolfgang (1990): Die real existierenden grammatischen Ellipsen und die Norm. Eine Bestandsaufnahme. In: Sprachwissenschaft 15, 241-366.

Nichols, Johanna (1986): Head-marking and dependent-marking grammar. In: Language 62, 56-119.

Nichols, Johanna/Woodbury, Anthony C. (Hg.) (1985): Grammar inside and outside the clause. Some approaches to theory from the field. Cambridge et al.

Olsen, Susan/Fanselow, Gisbert (1991): DET, COMP und INFL: Zur Syntax funktionaler Kategorien und grammatischer Funktionen. In: Olsen/Fanselow (Hg.) (1991) $1-14$.

Olsen, Susan/Fanselow, Gisbert (Hg.) (1991): „DET, COMP und INFL“. Zur Syntax funktionaler Kategorien und grammatischer Funktionen (Linguistische Arbeiten 263). Tübingen.

Pasierbsky, Fritz (1981): Sprachtypologische Aspekte der Valenztheorie unter besonderer Berücksichtigung des Deutschen. In: ZPSK 34, 160-177. 
Pütz, Herbert (1986): Über die Syntax der Pronominalform „es“ im modernen Deutsch (Studien zur deutschen Grammatik 3). 2., durchges. Aufl. Tübingen.

Reis, Marga (1982): Zum Subjektbegriff im Deutschen. In: Abraham (Hg.) (1982) $171-211$.

Rizzi, Luigi (1986): Null Objects in Italian and the Theory of pro. In: Linguistic Inquiry $17,501-557$.

Safir, Ken (1985): Missing Subjects in German. In: Toman (Hg.) (1985) 193-229.

Schumacher, Helmut (1988): Valenzbibliographie. 2., erw. und verbess. Aufl. Mannheim.

Schwitalla, Johannes (1985): Verbvalenz und Text. In: DaF 22, 266-270.

Schwitalla, Johannes (1988): Kommunikative Bedingungen für Ergänzungsrealisierungen. In: Helbig (Hg.) (1988) 74-84.

Seiler, Hansjakob/Stachowiak, Franz Josef (Hg.) (1982): Apprehension: Das sprachliche Erfassen von Gegenständen. Teil II: Die Techniken und ihr Zusammenhang in Einzelsprachen (LUS 1/II). Tübingen.

Seyfert, Gernot (1976): Zur Theorie der Verbgrammatik (Tübinger Beiträge zur Linguistik 73). Tübingen.

Shin, Soo Song (1988): Zur leeren Kategorie des Deutschen. In: Zeitschrift für Sprachwissenschaft 7, 60-91.

Shopen, Timothy (Hg.) (1985): Language typology and syntactic description. Band 1: Clause structure. Cambridge et al.

Stechow, Arnim von/Sternefeld, Wolfgang (1988): Bausteine syntaktischen Wissens. Ein Lehrbuch der generativen Grammatik. Opladen.

Stickel, Gerhard (Hg.) (1984): Pragmatik in der Grammatik. Jahrbuch 1983 des Instituts für deutsche Sprache (Sprache der Gegenwart 60). Düsseldorf.

Tesnière, Lucien (1959): Eléments de syntaxe structurale. Paris.

Toman, Jindřich (Hg.) (1985): Studies in German Grammar (Studies in Generative Grammar 21). Dordrecht.

Ulbricht, Adelheid (1987): Subjektreduzierungen - eine Fehlerquelle bei Deutsch lernenden Ungarn. In: DaF 24, 172-176.

Valin, Robert D. van (1985): Case marking and the strukture of the Lakhota clause. In: Nichols/Woodbury (Hg.) (1985) 363-413.

Wegener, Heide (1990): Komplemente in der Dependenzgrammatik und in der Rektionsund Bindungstheorie. Die Verwendung der Kasus im Deutschen. In: ZGL 18, $150-184$.

Weingartner, Paul/Czermak, Johannes (Hg.) (1983): Erkenntnis- und Wissenschaftstheorie. Akten des 7. Internationalen Wittgenstein Symposiums. Wien.

Weinrich, Harald (1967): Syntax als Dialektik (Bochumer Diskussion). In: Poetica 1, 109-126.

Weinrich, Harald (1982): Textgrammatik der französischen Sprache. Stuttgart.

Welke, Klaus (1992): Theorie der Dependenz und Valenz. [erscheint]

Adresse des Verfassers: Dr. Vilmos Ágel, Deutsches Institut Loránd-Eötvös-Universität Budapest, Ajtósi Dürer sor 19-21, H-1146 Budapest. 